University of Redlands

\title{
Spatial Representation of NOAA's Remotely Operated Vehicles (ROVs) Dive Tracks
}

\author{
A Major Individual Project submitted in partial satisfaction of the requirements \\ for the degree of Master of Science in Geographic Information Science \\ by \\ Moses Kamau Thiong'o \\ Douglas Flewelling, Ph.D., Committee Chair \\ Mark Kumler, Ph.D.
}

January 2020 
Spatial Representation of NOAA's Remotely Operated Vehicles (ROVs) Dive Tracks

Copyright (C) 2020

by

Moses Kamau Thiong'o 
The report of Moses Kamau Thiong'o is approved.

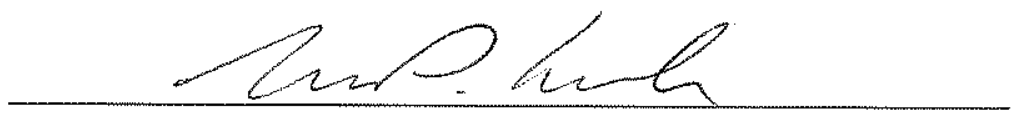

Mark Kumler, Ph.D.

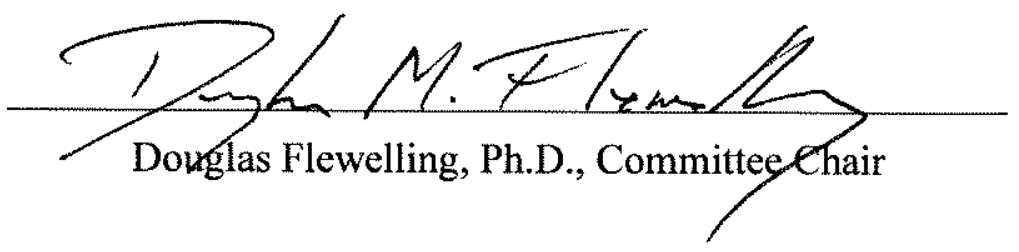

January 2020 



\section{Acknowledgements}

First and most importantly, I reserve this first paragraph for my Almighty God. As in Proverbs 3:6, I ought to acknowledge Him always, since He hath maketh all my paths straight. I thank God for my eyes which daily embrace heaven and earth to the point of making it this far. I persevered and with His gift of wisdom, strength, good health, peace of mind and passion, I was able to successfully complete my studies.

Secondly, I'd like to thank everyone who helped me in any way because this listing will not be able to include everyone. Going forward, while the term MIP, where "I" stands for Individual, was the chorus for the past whole study period, it would not have been possible to achieve without the hands of many. I'd like to extend heartfelt and sincere gratitude to my able advisor and committee chair, Professor Douglas Flewelling, for his invaluable contributions and his positive critiques and counsel towards my success. He was one of my closest faculty members and this was attributed to his neverending smile. Professor Mark Kumler, with his expertise in his area of specialty, projections, was my eye opener. Through his lessons, I was able to make stunning maps of Africa in a globular perspective. Special thanks goes to Professor Fang Ren, the Department Chair, for being there always to respond to any challenge that I came across when handling any subject in her area of expertise. Special thanks goes to Professor Ruijin Ma for bestowing me with knowledge of Remote Sensing and Python programming, which I will use my entire life. Also, it would be very unjust to leave behind Andrea Alvarado who was always there, often behind the scenes, to ensure everything went well in the department of Geographical Information Science while at the University of Redlands.

Thirdly, I'd like to sincerely thank Jack and Laura Dangermond for sponsoring every facet of my studies. My studies would have been a monster for without your priceless help. God bless you always and may Esri Inc. blossom even in other planets.

To my family headed by Paul and Anne Thiong'o and my siblings in the Republic of Kenya, my studies would not have been actualized without your prayers. You constantly called me to ensure that I was safe and had eaten something each day. My success is your success.

Here is a message for you that I wrote in Swahili language - Baba na mama, nyie mlonizaa, mlitaabika mno kwa nguvu mlokuwa nazo ili mie mwanenyu nikipate kisomo. Letu kubwa lilikuwa bora ni afya kuliko mali. Sikuyataka makubwa kama walokuwanayo mashoga wangu, ila mie hapa, nilitaka mapenzi yenu pekee. Hamkuninyima wala kunipimiya. Baba na mama, dua zenu zishatimika. Kwake Rabuka, nawaombeeni mpate heri na afya njema siku zote. Nawashukuru sana! Ngā̄ wa mūoyo aromūrathima hingo ciothe rīrīa mwì thì ìno. Nìnie kīhī kīanyu kīrīa gīkūrū, Thai!

“Guoya ūtūūragia ūkīa mūcī̄”- Kikuyu Proverb!

Literal translation: The fear (of toil) keeps your house poor.

-Kamau wa Thiong'o (Nya-mūriū). 



\begin{abstract}
Spatial Representation of NOAA's Remotely Operated Vehicles (ROVs) Dive Tracks

by

Moses Kamau Thiong'o

The oceans make up about $70 \%$ of the earth's surface and serve as habitats for many deep and shallow creatures. In depths of about 50 meters and more, deep-sea corals and sponges occur mostly along seamounts, continental margins, undersea canyons and ridges. They, deep-sea corals and sponges, play a key role in supporting the health of the ocean as they preserve the biodiversity and long-term sustainability of commercial and recreational fish species. With the many benefits that are attached to deep-sea corals and sponges, the Deep-Sea Corals and Research Technology Program (DSCRTP) has been collecting coral and sponge location data from hundreds of remotely operated vehicle (ROV) surveys. However, DSCRTP does not have a spatial representation of the area covered by each ROV while searching for corals and sponges in the deep-sea. A spatial representation would provide critical information to researchers and managers to understand where a survey for corals and sponges has happened, and where a survey is yet to be done in the deep-sea. Therefore, the goal of this study is to create a spatial representation of the ROV surveys that have been collected in Monterey Bay and Hawaii sections of the deep-sea.
\end{abstract}





\section{Table of Contents}

Chapter 1 - Introduction .................................................................................................. 1

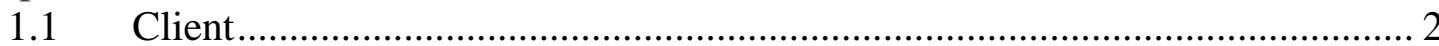

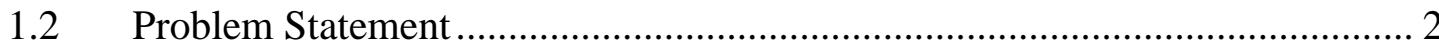

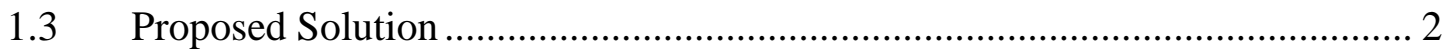

1.3.1 Goals and Objectives ............................................................................ 2

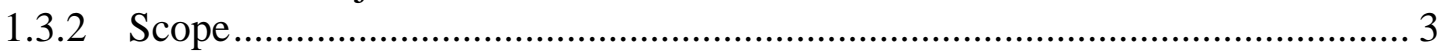

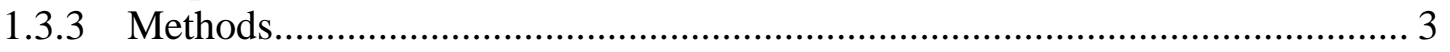

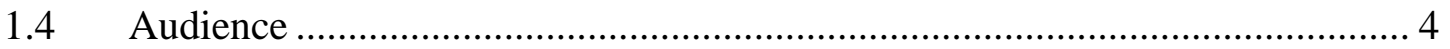

1.5 Overview of the Rest of this Report ................................................................ 4

Chapter 2 - Background and Literature Review ................................................................ 5

$2.1 \quad$ Deep-sea Corals and Sponges.................................................................. 5

2.2 Coral and Sponge Mapping and Representation............................................. 5

2.2.1 Methods of Representing an Area Around Points ........................................... 6

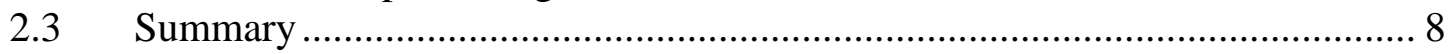

Chapter 3 - Systems Analysis and Design..................................................................... 9

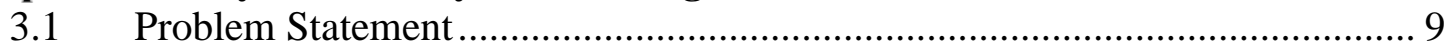

3.2 Requirements Analysis ........................................................................ 9

3.2.1 Functional Requirements .................................................................... 9

3.2.2 Non-Functional Requirements ............................................................... 10

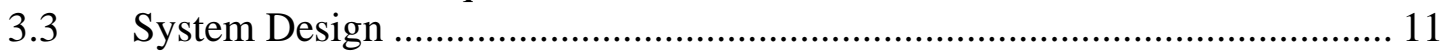

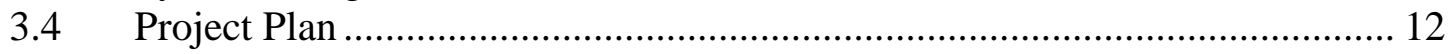

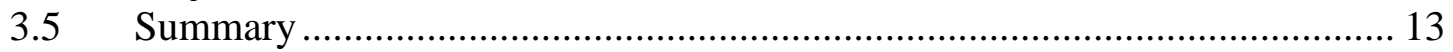

Chapter 4 - Database Design................................................................................................... 15

4.1 Conceptual Data Model ........................................................................ 15

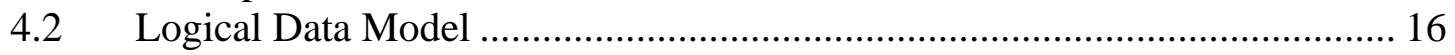

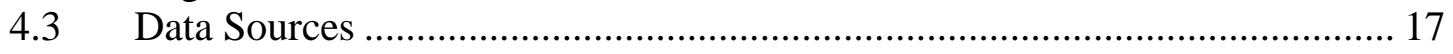

4.4 Data Scrubbing and Loading …………………...................................... 19

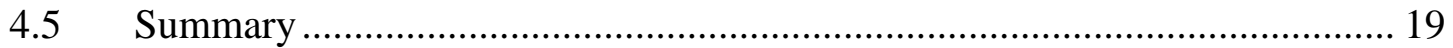

Chapter 5 - Implementation.............................................................................................. 21

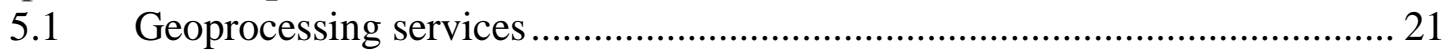

5.1.1 Add Event Number Model Tool .................................................................... 21

5.2 Options for ROV Surveyed Areas Representation ......................................... 22

5.2.1 The Points To Line Approach...................................................................... 23

5.2.2 The Aggregating Points Approach................................................................ 24

5.2.3 The Buffer Approach ................................................................................... 25

5.3 Realization of the ROV Survey Area Generator Tool ..................................... 26

5.3.1 Analysis and Output of the ROV Survey Area Generator Tool ..................... 30

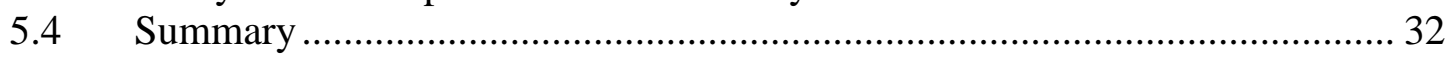

Chapter 6 - Results and Analysis..................................................................................... 33

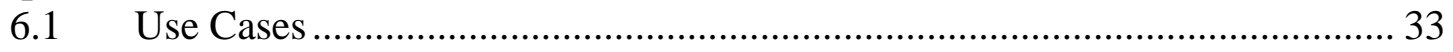

6.1.1 Deep-sea Mining Activities ........................................................................... 33 


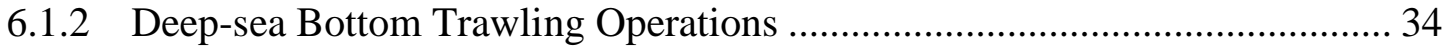

6.1.3 Other Applications of the Project ................................................................ 35

6.2 The Analysis and Results of the ROV Survey Area Generator Tool ............. 35

6.3 Representation Complications and Data Issues .......................................... 38

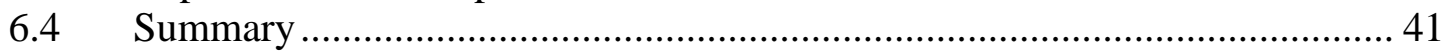

Chapter 7 - Conclusions and Future Work .................................................................. 43

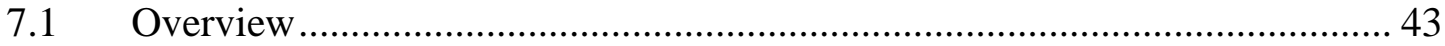

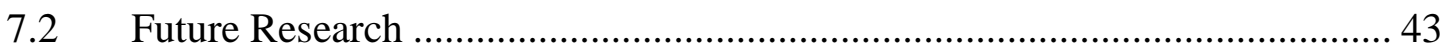

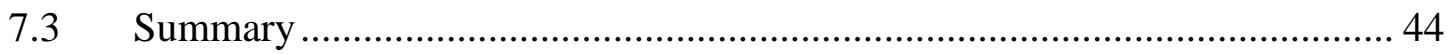

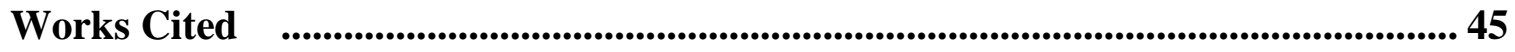

Appendix A. Project Models ................................................................................................ 47

Appendix B. List of Grouping Fields for the Remotely Operated Vehicles

Observations $\quad$..................................................................................................................................... 48 


\section{Table of Figures}

Figure 1-1: A glass sponge containing cephalopod eggs. Photo: NOAA Okeanos Explorer Program/2013 Northeast U.S. Canyons Expedition ................................. 1

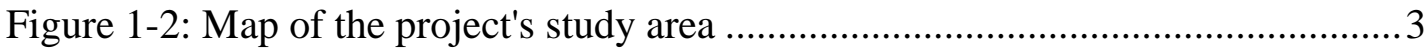

Figure 2-1: NOAA's Deep-Sea Coral and Sponge Map Portal................................. 6

Figure 2-2: Regular tiling, a) Triangular tiling, b) Hexagonal tiling and c) Square tiling, showing the three representations of dive track 957 .................................. 7

Figure 2-3: The a) Voronoi and b) Convex Hull computation Geometries ................. 7

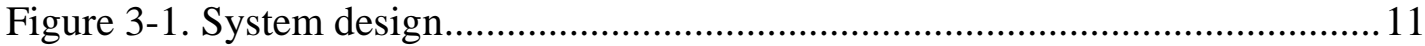

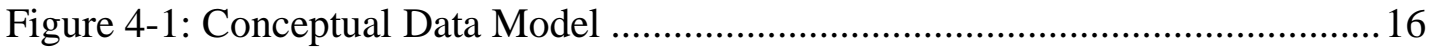

Figure 4-2: Logical database schema................................................................ 17

Figure 4-3: The Attribute rows of Remotely Operated Vehicles Dive observations

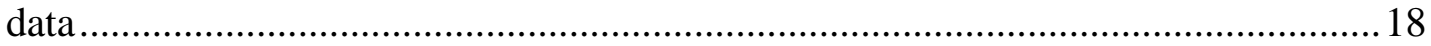

Figure 4-4: The ROVs used to collect the dive tracks observations ........................ 19

Figure 5-1: The Null observations that were not used to produce areas surveyed in

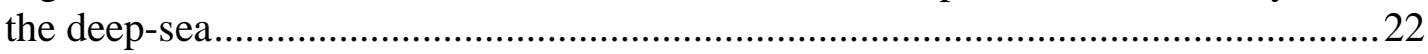

Figure 5-2: Add Event Number Tool User Interface .................................................22

Figure 5-3: An illustration of How Points To Line tool is supposed to work. (Source:

Esri Points To Line tool documentation) ............................................................23

Figure 5-4: The Points To Line tool in ArcGIS Pro showing the parameters that the

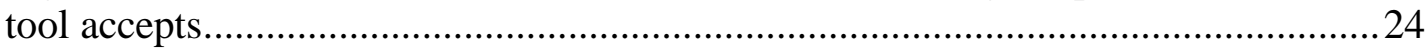

Figure 5-5: Example of the output of Points To Line tool........................................24

Figure 5-6: Example of the output of Aggregate Point tool from GeoAnalytics Server toolbox with each square measuring 500 meters by 500 meters ............................25

Figure 5-7: An illustration of how Buffer tool is supposed to work. (Source: Esri

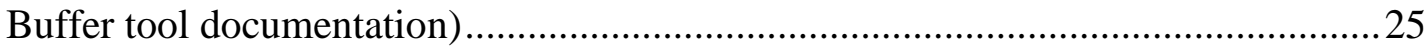

Figure 5-8: Example of Buffer tool output produced using a buffer of 100 meters .. 26

Figure 5-9: ROV Survey Area Generator ModelBuilder.......................................2 27

Figure 5-10: The ROV Survey Area Generator Tool .............................................. 28

Figure 5-11: A view of the attribute table for ROV spatial representation ................29

Figure 5-12: Dive 80 showing dives with the same EventNo but recorded in different

years. This shows why grouping was vital to avoid such issues. ............................30

Figure 5-12: A section of ROV coverages in Monterey Bay .................................. 31

Figure 5-13: A section of ROV coverages in Monterey Bay superimposed with ROV

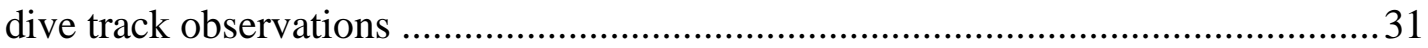

Figure 5-14: ROV representation of a dive near Hawaii ........................................ 32

Figure 6-1: Schematics of deep-sea mining process................................................. 34

Figure 6-2: A comparison of coverage areas in a section Monterey Bay showing the usage of the Aggregate Points, Minimum Bounding Geometry and Buffer

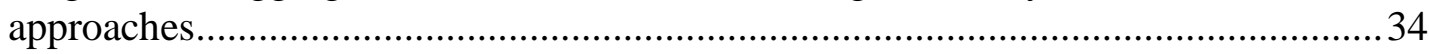

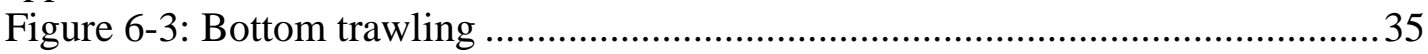

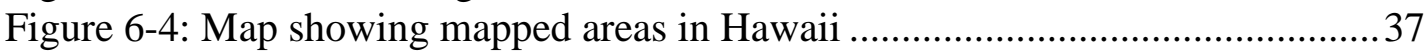

Figure 6-5: Map showing mapped areas in Monterey Bay ........................................ 38

Figure 6-6: Using the Points To Line Approach....................................................... 39 
Figure 6-7: Using the Aggregate Points approach ................................................ 39 Figure 6-8: Superimposition of Minimum Bounding Geometry and Aggregate Points

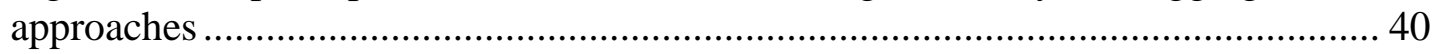

Figure 6-9: Using the Minimum Bounding Geometry approach ........................... 40 


\section{List of Tables}

Table 1. Summary of Project Functional Requirements ...................................... 10

Table 2. Summary of Project Non-Functional Requirements................................ 11

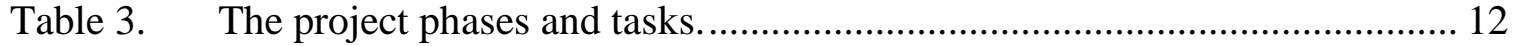

Table 4. The fields used to group the ROV data points ............................................ 29

Table 5. Performance results of the ROV Survey Area Generator Tool ..................... 36

Table 6. Mapped Areas of ROV using the ROV Survey Area Generator Tool and

Aggregate Points in GeoAnalytics Desktop toolbox approaches .................................. 41 



\section{List of Acronyms and Definitions}

DSCRTP Deep-sea Corals and Research Technology Program

MBARI Monterey Bay Aquarium Research Institute

MSA Magnuson Stevens Act

NOAA National Oceanic and Atmospheric Administration

ROV Remotely Operated Vehicle

UTM Universal Transverse Mercator

WGS World Geodetic System 



\section{Chapter 1 - Introduction}

Deep-sea corals and sponges are the most important biogenic habitats in the deep-sea. They are associated with many benefits such as acting like a reference for climate records whereby, for example, the composition of trapped oxygen is assessed to approximate seasonal temperatures and rainfall and to create a record of change over time. Figure 1-1 shows a glass sponge with hatching celaphopod eggs. This project aims at spatially representing the survey using Remotely Operated Vehicles (ROV) dive tracks as polygons of areas where exploration for deep sea corals and sponges has taken place. National Oceanic and Atmospheric Administration's (NOAA's) Deep-sea Corals and Research Technology Program (DSCRTP) would like to have a representation of the areas mapped since it would help in accounting for a measure of effort and putting the number of animals found in each location into more comparable context. In return, the resource managers and other regional fishery management councils would be able to determine rules governing where, when, and how people can fish the deep sea, and protect the corals and sponges. The managers would also be able to administer permits for exploitation of areas of the deep-sea depending on whether those areas have been mapped or not and whether the density of corals and sponges is high or low.

In this chapter, the client for the project, the definition of the problem being addressed, the solution proposed, the goals and objectives as well as the scope, the methods and audience are discussed.

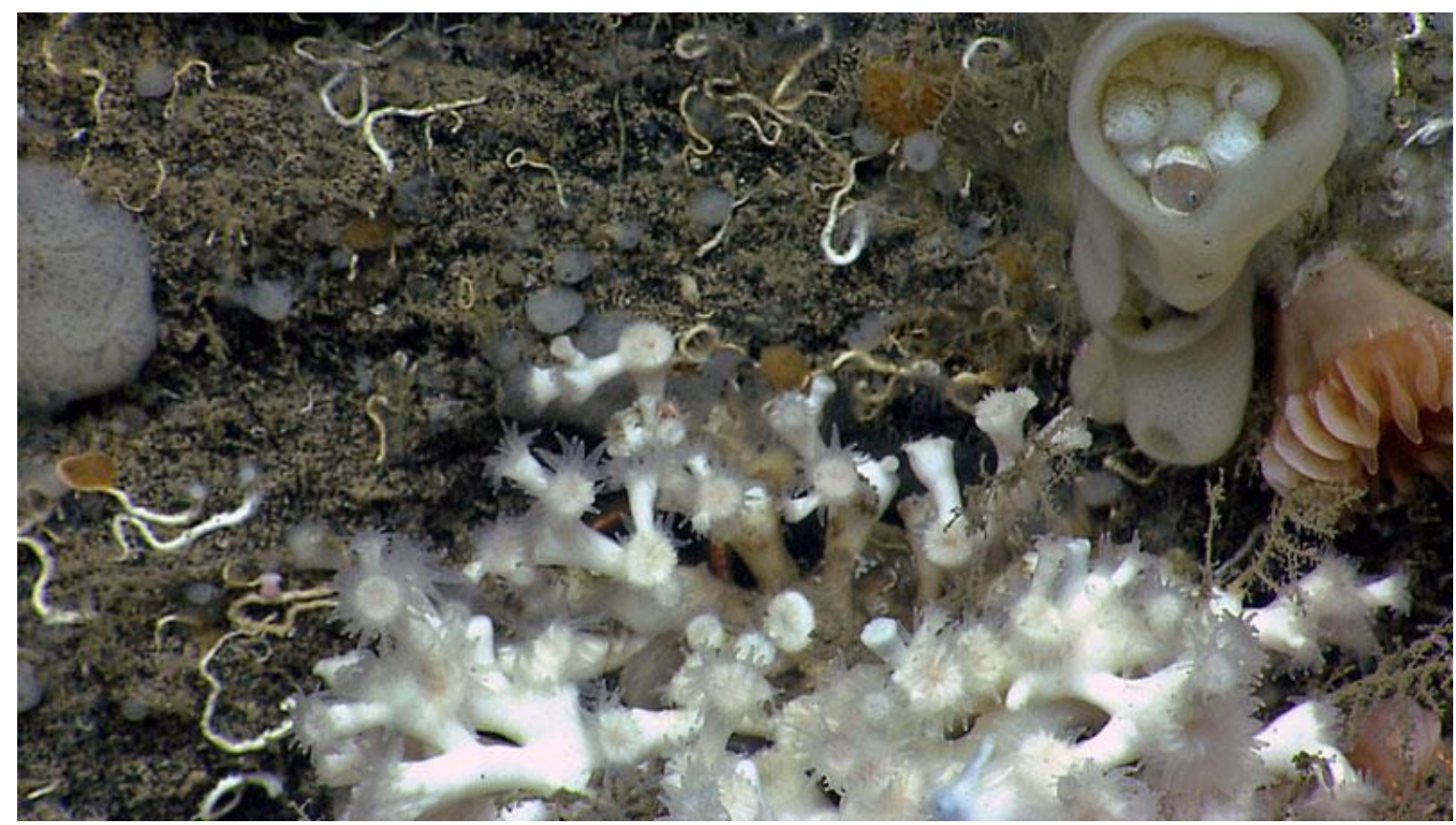

Figure 1-1: A glass sponge containing cephalopod eggs. Photo: NOAA Okeanos Explorer Program/2013 Northeast U.S. Canyons Expedition 


\subsection{Client}

The client for this project was the National Oceanic and Atmospheric Administration's (NOAA's) DSCRTP with Heather Coleman and Matt Dornback acting as the point of contact. The DSCRTP studies and provides scientific information required for the conservation and management of the deep-sea corals and sponge systems in the United States (About Us-NOAA's Deep-Sea Coral Data Portal, 2017). The client's roles included: guidance in defining the scope of the project, analysis of the project requirements, and provision of required datasets, as well as giving feedback to the developed solution.

\subsection{Problem Statement}

The client wanted to produce a GIS representation of the ROV dive tracks as polygons showing the areas that have been mapped in search for deep sea corals and sponges. A representation using polygons would help in accounting for a measure of effort and putting into context the areas that have been mapped thus making it easy to quantify the covered and uncovered areas. Having such a representation was a challenge for the client since the only representation that was present was discrete points of the dive tracks representing observations of the ROVs used. Representing the areas that have been mapped as polygons would be crucial in helping researchers and managers understand where corals and sponges have been looked for and not found and where a survey has not been done in the deep sea.

\subsection{Proposed Solution}

In order to provide a solution to the above problem and ensure a repeatability of generating such representations in future, a geoprocessing tool had to be customized using the existing functionality of tools in ArcGIS Pro Toolbox. The tool would run in ArcGIS Pro and allow the user to input a set of ROV dive track point features using the minimum bounding geometry tool, specify the grouping fields to be used in defining a group of dive tracks and make a buffer based on the input of the user. The output of the tool would be a bounding rectangle showing the ROV's coverage area in the deep-sea.

\subsubsection{Goals and Objectives}

The main goal of the project was to spatially represent the areas covered by ROVs during a search for corals and sponges. This would help in putting the number of animals found in each location into more comparable contexts, thus assisting researchers and managers to understand where corals and sponges have been looked for and not found, and areas which are yet to be surveyed in the deep-sea. The main objective of this study was to develop a custom geoprocessing tool which would enhance repeatability in production of representations of mapped areas during a search for corals and sponges in the deep-sea. 


\subsubsection{Scope}

The study area of this project spread from Seattle, the Monterey Bay, Los Angeles, Gulf of California and Hawaii sections of the deep-sea as shown in Figure 1-2. The areas are situated in the Pacific Ocean on the west coast of the United States. The main deliverable of this project was the representation of the areas that have been surveyed using ROVs. This would be accomplished through creating a customized geoprocessing tool that would help in showing the footprints of ROV surveys which had already been carried out in the deep-sea.

Due to their depths and big distances from the shores, deep seas have not been well explored compared to the shallow seas ecosystems, which have been well observed and experimented (Jamieson, 2015). The individual ROV surveyed areas for this study were represented as points. This had no consideration of the area viewed by the camera system mounted on the ROV. A more generic representation of the dive points would be a line but for this project, the best representation, and which was the main deliverable, were polygons that represented the areas that had been mapped in the deep-sea in efforts for searching for new and existing corals using ROVs. This goal was achieved by creating a custom geoprocessing tool in ArcGIS Pro that would help in making an output of the surveyed areas.

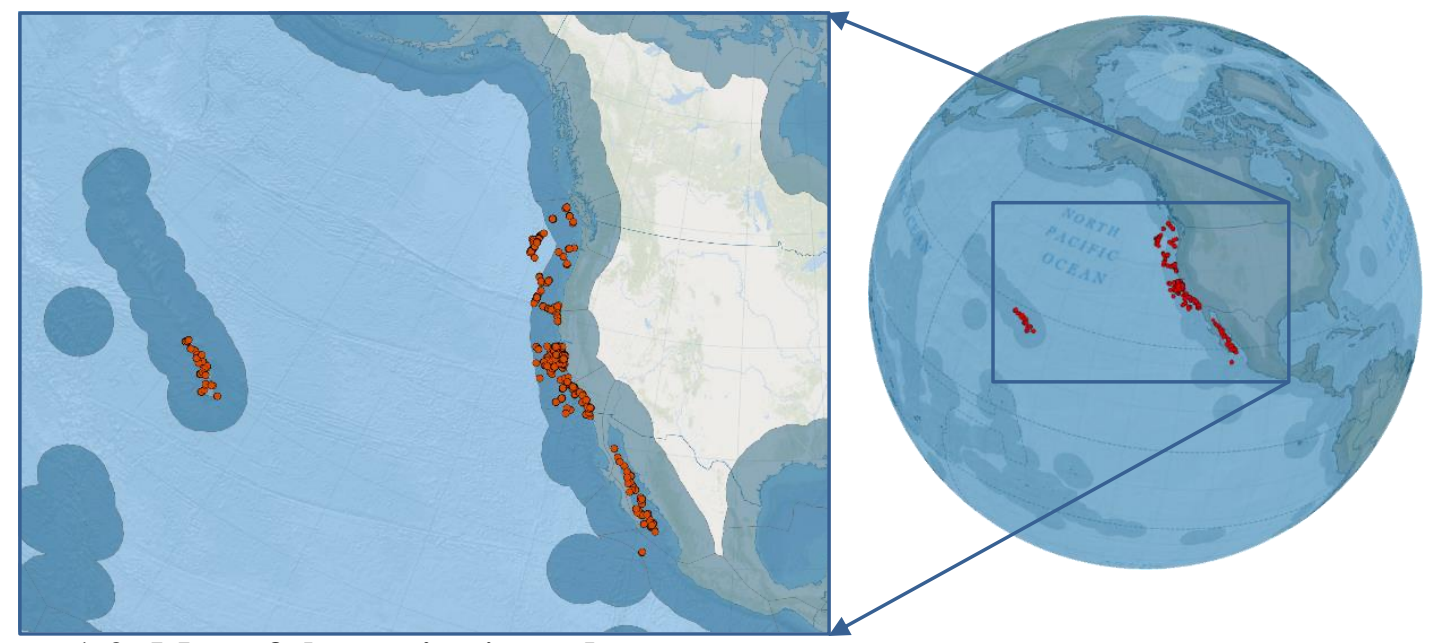

Figure 1-2: Map of the project's study area

\subsubsection{Methods}

The Monterey Bay Aquarium ROV dive tracks data was used for this project. The client provided the data in the comma separated value (CSV) format. This data was loaded into ArcGIS Pro version 2.4.1 and projected with World Geodetic System Datum 1984 (WGS84). To accomplish the tasks of this project, geoprocessing tools were developed by customizing the minimum bounding geometry and buffer tools in the ArcGIS Toolbox. 


\subsection{Audience}

The main target for this project are the fisheries management councils, ocean conservation organizations that are involved in the conservation of the marine biodiversity and the professionals in the GIS and development field who may consider taking the project further. Other target audiences are the groups involved in any kind of maritime activity such as deep-sea mining, fishing, and mapping (e.g. the NOAA ship Okeanos Explorer - United States of America's deep-sea exploration ship).

\subsection{Overview of the Rest of this Report}

The rest of this report is a detailed discussion about the representation of the areas mapped in the deep-sea using ROVs. In Chapter 2, the background of the deep-sea corals and sponges is given. Also, the mapping of the corals and the sponges is discussed. Chapter 3 discusses the system analysis and design, the problem statement, analysis of the requirements, and project design. Chapter 4 details the conceptual model and database design implemented for this project. The implementation process of the of the project is addressed in Chapter 5 and the results and the use cases of the project are presented in Chapter 6. Finally, Chapter 7 presents a summary of the project and possible GIS future work related to this project. 


\section{Chapter 2 - Background and Literature Review}

In this chapter the relevant topics regarding the mapping of deep-sea corals and sponges are discussed. Section 2.1 discusses deep-sea corals and sponges. Section 2.2 reviews coral and sponge mapping and representation and 2.3 gives a summary of the entire chapter.

\subsection{Deep-sea Corals and Sponges}

The deep-sea corals and sponges occur throughout the world's seven seas (Hourigan et $a l .$, 2017). These seas, as indicated by Hovland (2008), have become a new area of research since they offer new exciting frontiers of exploration with the help of modern advanced range of methods like remote control surveying and documentation. On the other hand, scholars (Henry \& Roberts, 2007; Hourigan et al., n.d.) have reported that deep-sea corals and sponges make a crucial biogenic environment within the deep sea and therefore, ecosystems of unbelievable variety and biodiversity thrive here very well. Mehbub et al. (2014), reviewed that about $29 \%$ of the discoveries in marine ecosystems were natural products derived from sponges.

The deep-sea corals are portrayed as critical regions of biodiversity hotspots (Henry \& Roberts, 2007). Nevertheless, as Parrish et al. (2007) put it, a significant variety of species are still undescribed in the United States' waters. Unlike corals, Hogg et al. (2010), reported that the US deep water sponges' biodiversity have not been catalogued systematically and, most likely, the sponges' abundance exceeds that of corals. On top of this, Hogg et al., (2010) and Leal, et al., (2012) demonstrated that deep-sea corals and sponges are valuable to humans in several ways; they act as sources of aquatic natural products that may be crucial to the development of new drugs to treat human ailments. In addition, as corals and sponges grow and enlarge in size, they produce habitats for a host of different types of species like sea mammals, fish and invertebrates (Hourigan et al., 2017).

\subsection{Coral and Sponge Mapping and Representation}

In a GIS setting, events or objects in the actual world can be recorded in either 2- or 3dimension space and they need to be reduced into spatial entities (Gimond, n.d.). Following this, spatial entities can be represented as vector data model or a raster data model. The Deep-sea Corals and Research Technology Program (DSCRTP) records the location of observation as points (https://www.ncei.noaa.gov/maps/deep-seacorals/mapSites.htm), one of the three geometric primitives of a vector feature. Figure 21 shows the NOAA's Deep-Sea Coral and Sponge Map Portal. Every point is characterized by one pair of coordinates, which represents a specific location in a coordinate system. For vector features (points, polylines, polygons), points are the most basic since they lack length and area. On a map, they can be represented as a circle, square or any other sign, like a plus sign. Area cannot be computed for points and therefore, to reduce the ambiguity of representation, points can be represented as polylines or polygons through various means such as performing a buffer analysis. 


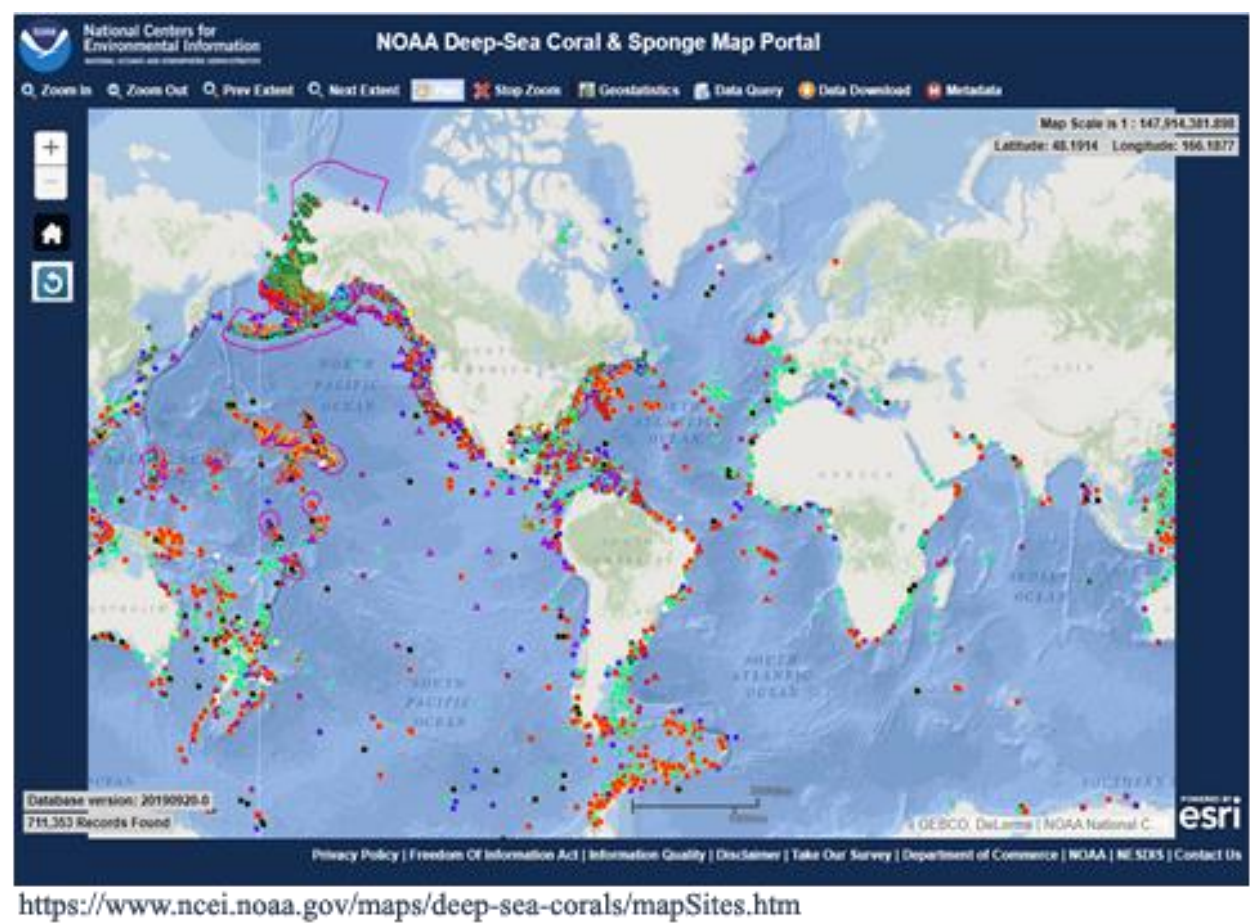

Figure 2-1: NOAA's Deep-Sea Coral and Sponge Map Portal

In contrast, various methods in the past have been applied in mapping the existence of corals and sponges; remote sensing (Mumby, et al., 1997) and multibeam sonar and ROV video observations (Neves et al., 2014) are some of the methods that have been used widely. Nonetheless, due to the oceans' expansiveness, much of the deep-sea corals and sponges have been scantily mapped to document the information about them (Osundwa, 2016), and there are a lot of information gaps for areas that are not mapped.

As an effort to achieve more in ocean mapping, NOAA's DSCRTP, established in 2009 with the reauthorization of the Magnuson-Stevens Act (MSA), has been mapping the deep-sea corals and sponges for depths below 50 meters (Hourigan et al., 2015), where most of the corals and sponges occur, using the ROVs (Remotely Operated Vehicles). ROVs can reach depths which are not easy for a person to reach, thus making them one of the greatest sources of collecting deep-sea information on the condition of deep-sea corals and sponges, which are the building blocks of the seafloor. This documentation is ideal since it would help increase protection efforts.

\subsubsection{Methods of Representing an Area Around Points}

Several studies have been conducted utilizing the capabilities of GIS in deep-sea corals and sponges mapping. To illustrate this, Neves, Du Preez, \& Edinger (2013), have utilized the multibeam sonar and ROV video observations in mapping coral and sponge habitats on a shelf-depth environment. The trio indicated that mapping the habitats of deep-sea coral and sponge is crucial as it helps in the effective management and conservation of these vulnerable marine ecosystems.

The ROVs (Huvenne et al., 2016) have been used in assessing the recovery and status of the fisheries and Marine Protected Areas (MPAs). They are ideal, as scuba 
divers and trawlers cannot get beyond certain depths in the deep-sea. Following this, Gori et al. (2011), show the advantage of ROVs since they have helped in making thorough observations as well as quantitative sampling in the recent past. There are many methods which have been implemented with an aim to map the corals and sponges. Osundwa (2016), developed a coral reef mapping tool in which he was utilizing Volunteered Geographic Information (VGI) Goodchild (2007).

Another approach is the use of tiling. Several scholars have discussed various methods which could be used to represent an area as mapped or not. For example, Grunbaum \& Shephard (1977), have discussed at length about tiling or tessellation (from tiles). The duo mention that tessellation covers a surface with neither gaps nor overlaps. Regular tiling has been in use since antiquity. They are easily accessible and have received a good mathematical research. Grunbaum \& Shephard (1977), state that a regular convex polygon with $n$ sides is named " $n$-gon" with a symbol $\{n\}$ (p. 227). What this means is that, $\{3\},\{4\},\{6\}$ represents an equilateral triangle, square, a regular pentagon, and regular hexagon respectively. Figure 2-2 shows the three types of regular tiling as discussed above. According to Chavey (1989), many theorems of classification using highly symmetrical tiling by regular polygons were developed in the 1960s.
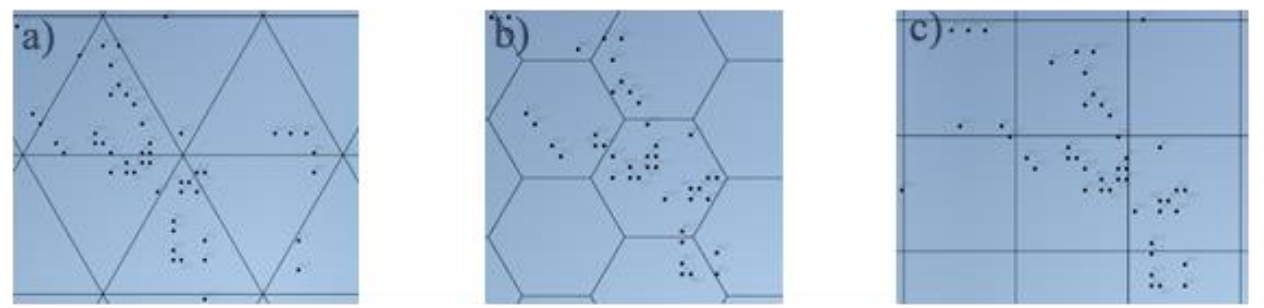

Figure 2-2: Regular tiling, a) Triangular tiling, b) Hexagonal tiling and c) Square tiling, showing the three representations of dive track 957

Minimum Bounding Box (Convex Hulls) could also be used to show the smallest possible area encompassing the dive tracks. According to van Kreveld (2006), there are a variety of computation geometry including: Convex hulls, Line segment, Voronoi diagrams (Thiessen polygons), and Delaunay triangulation. Convex hulls have been used in different fields, such as zoology, to represent the smallest area for a set of points in two dimension (2D) (Cadenas \& Megson, 2019). To map the spread of points using the convex hulls, highly dispersed points lead to a large uncovered space to be represented as mapped which is not the case. Figure 2-3 shows two computation geometries done in ArcGIS Pro.
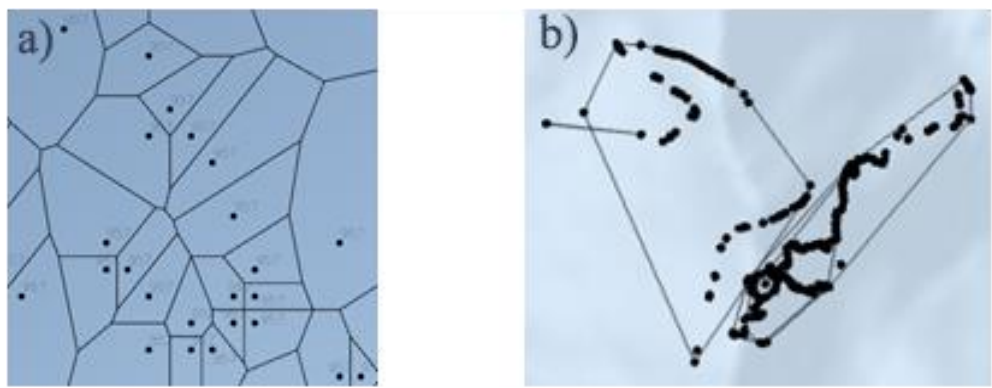

Figure 2-3: The a) Voronoi and b) Convex Hull computation Geometries 
On the other hand, various research has been done on buffer analysis. In the context of GIS, buffer analysis entails making a zone or zones of specified distance(s) around a feature(s) (Hehai, 1997) and (Zhou et al., 2018). Also, it has been stated (Peng Dong, et $a l ., 2003)$ that buffer analysis is an imperative function for spatial analysis. Nevertheless, in spite of Zhou et al. (2018), saying that buffer analysis helps make complex problems more scientific and visually appealing, Peng Dong et al. (2003), demonstrates that buffer analysis has issues due to self-intersections, island polygon problems and the overlapping polygons.

\subsection{Summary}

Notable research has been done on the benthic environment in efforts to unearth information on corals and sponges. Corals and sponges occur at the bottom of the ocean. In this chapter, topics on mapping of the deep-sea corals and sponges have been reviewed. Also, the methods available for representing points as areas were each discussed to help discover the potentiality of each of them. The methods addressed above helped to decide which representation methods of the dive tracks in the deep-sea were best. 


\section{Chapter 3 - Systems Analysis and Design}

Chapter 3 discusses the analysis of the client's requirements as well as the design of the proposed solution. The requirements; which are categorized into functional and nonfunctional requirements of the system design, are discussed and, the problem statement is revisited. Analysis of the stages of the project from the initial to closure are also reviewed.

\subsection{Problem Statement}

The deep-sea corals and sponges are one of the most vital biogenic habitats in the deepsea. The Deep-sea Corals and Research Technology Program (DSCRTP) has been exploring the deep seas using the NOAA ship Okeanos in efforts to search for new and existing corals. The information that is collected for corals and sponges using the ROVs is currently recorded as discrete points. The DSCRTP had difficulty in representing the ROV dive tracks of areas they have surveyed for corals and sponges. They needed a more representative way using polygons instead of discrete points to show the sections of the deep-sea which they have been able to cover. With such a representation that the client wanted to achieve, it would be easier for them to make decisions on how the deep-sea was to be exploited and the new areas that needed mapping to establish the density of corals and sponges.

\subsection{Requirements Analysis}

This project was heavily dependent on an understanding and analysis of its requirements in order to achieve the end goal, making a representation of the areas that had been surveyed in the deep-sea using ROVs. This project included the functional and nonfunctional requirements. First, the functional requirements details the system's functionality that would be provided to the end user; they included the tasks that the user could do and the resulting information that would be obtained. Secondly, the nonfunctional requirements outline the way a system should be functioning by referring to the tools and the methods so that the end goals and objectives can be realized. For this project, the requirements needed to be traceable, consistent, complete, unambiguous and acceptable by the client. The functional requirements were to be achieved using custom geoprocessing tools that were created in ArcGIS Pro version 2.4.1.

\subsubsection{Functional Requirements}

The functional requirements were the capabilities that the geoprocessing tools and models created would function properly as per the needs of the client. They would ensure that the tools worked properly to produce the required results. Table 3 gives the description of each project's functional requirement. 
Table 1. Summary of Project Functional Requirements

\begin{tabular}{|c|c|}
\hline Requirement & Requirement Description \\
\hline $\begin{array}{l}\text { Input ROVs } \\
\text { Parameter }\end{array}$ & $\begin{array}{l}\text { This is a required parameter. ROV dive tracks are usually recorded as } \\
\text { discrete points during an exploration. Therefore, the input shall be the } \\
\text { points/multipoint. The data type for this parameter will be feature } \\
\text { layer. }\end{array}$ \\
\hline $\begin{array}{l}\text { Geometry } \\
\text { Type }\end{array}$ & $\begin{array}{l}\text { This will specify what type of minimum bounding geometry the } \\
\text { output polygons will represent. The project will implement } \\
\text { RECTANGLE_BY_AREA - The rectangle of the smallest area } \\
\text { enclosing an input feature. This will be the default type of the tool. } \\
\text { For this parameter, the data type will be a string. }\end{array}$ \\
\hline $\begin{array}{l}\text { Add Geometry } \\
\text { Attributes }\end{array}$ & $\begin{array}{l}\text { The parameter specifies whether to add the geometric attributes in the } \\
\text { output feature class or omit them in the output feature class. This will } \\
\text { be set to true so that geometric attributes will be added in the output } \\
\text { feature class. The data type of the parameter will be a Boolean. }\end{array}$ \\
\hline Group Option & $\begin{array}{l}\text { This parameter will specify how the input features will be grouped; } \\
\text { each group will be enclosed with one output polygon with LIST as } \\
\text { the grouping option. This will allow the input features to be grouped } \\
\text { based on their common values in the specified field or fields in the } \\
\text { group field parameter. The data type for this parameter will be string. }\end{array}$ \\
\hline Group Fields & $\begin{array}{l}\text { This parameter specifies the field or fields in the input features that } \\
\text { will be used to group features. Since a LIST will be specified as the } \\
\text { grouping option, at least one group field must be specified. All } \\
\text { features that have the same value in the specified field or fields will } \\
\text { be treated as a group. }\end{array}$ \\
\hline $\begin{array}{l}\text { Output } \\
\text { Parameter }\end{array}$ & $\begin{array}{l}\text { This is a required parameter. The output features of the tool will be } \\
\text { specified by the user to a local directory in the computer. }\end{array}$ \\
\hline Documentation & $\begin{array}{l}\text { A good tool is one which has a wealth of documentation so that the } \\
\text { user can understand how the tool functions. This tool will include } \\
\text { such documentation to guide the user. }\end{array}$ \\
\hline
\end{tabular}

\subsubsection{Non-Functional Requirements}

The non-functional requirements were the technologies needed to make sure that the functional requirements functioned properly. These requirements specifies both the hardware and system requirements of the machine that the user is required to have so that 
the ArcGIS Pro software can function properly. Table 4 shows the non-functional requirements of the project.

\section{Table 2. Summary of Project Non-Functional Requirements}

\begin{tabular}{|l|l|}
\hline Requirement & Requirement Description \\
\hline Operating System & $\begin{array}{l}\text { For the best functionality, it is highly recommended that } \\
\text { the user should use the latest versions of the Windows 10 } \\
\text { Home, Pro, and Enterprise (64 bit) is preferred for ArcGIS } \\
\text { Pro to work perfectly. }\end{array}$ \\
\hline Hardware & $\begin{array}{l}\text { The user's machine should have a RAM of } 8 \text { GB or } \\
\text { anything above that is the one which is recommended. A } \\
\text { minimum of 32 GB or more of free space on a solid-state } \\
\text { drive (SSD) is recommended as well. }\end{array}$ \\
\hline
\end{tabular}

\subsection{System Design}

Based on the understanding of the client's requirements, the system design was built based on the Esri ArcGIS Environment. The system comprised of two major components; data processing and analysis. The main deliverable of the project was Remotely Operated Vehicles (ROVs) coverage polygons based on the dive track points.

The system design starts with the ROV data which is in a csv format. The data was loaded into ArcGIS Pro using the XY Table to Point tool in the ArcGIS toolbox. In ArcGIS Pro, data cleaning was performed using the ModelBuilder tools and stored in a geodatabase. The cleaned data was used in the analysis to produce the minimum bounding rectangle to represent the areas that had been surveyed in deep-sea. The final surveyed polygons were then stored in a geodatabase in ArcGIS Pro. Figure 3-1 summarizes the system design of the project.

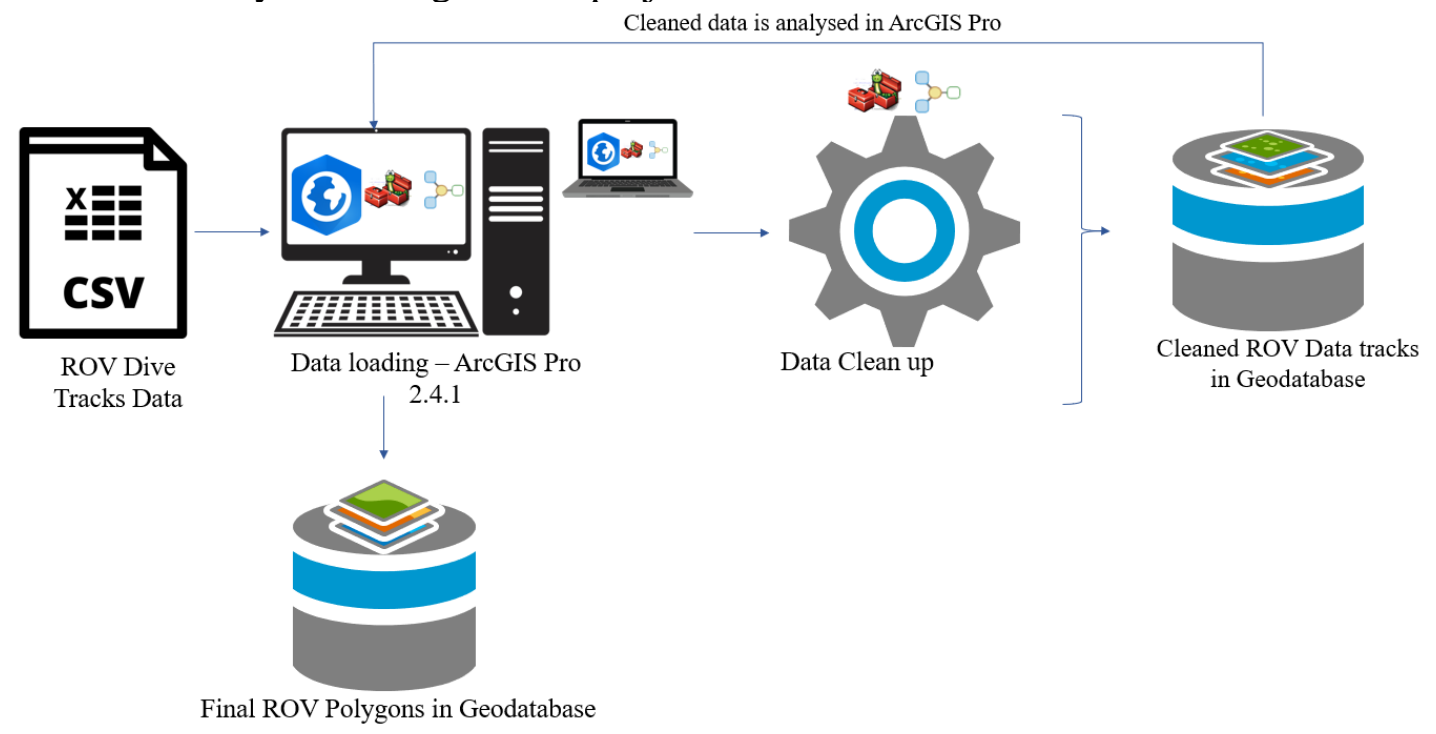

Figure 3-1. System design 


\subsection{Project Plan}

This project had four major phases; Planning, Design, Development and Testing, and Deployment. The planning phase was critical for this project since it involved the definition of the client's problem and the overall required product. The data, goals and the objectives of the project were provided by the client at the beginning. On the second phase, design, the necessary models and scripts were written to help in the process of data cleaning to make it ready for analysis. Data cleaning was essential since the after-analysis operations could not be done without a clean dataset. The third phase, development, comprised of tasks like creating custom geoprocessing tools which were to be run to produce the final product. Finally, deployment was the final phase where tasks such as quality control was undertaken. Table 5 below gives a more detailed work breakdown of the project's schedule.

Table 3. The project phases and tasks.

\begin{tabular}{|c|c|c|c|c|}
\hline $\begin{array}{l}\text { Sub- } \\
\text { section }\end{array}$ & Phase / Task Description & $\begin{array}{l}\text { Start } \\
\text { Month }\end{array}$ & End Month & $\begin{array}{l}\text { Labour } \\
\text { hours }\end{array}$ \\
\hline 1 & Plan & \multirow{5}{*}{$\begin{array}{l}\text { Feb. } \\
\text { Mar. } \\
\text { Mar. }\end{array}$} & \multirow{5}{*}{$\begin{array}{l}\text { Feb. } \\
\text { Mar. } \\
\text { Mar. }\end{array}$} & \multirow{3}{*}{20} \\
\hline 1.1 & $\begin{array}{l}\text { Understanding the Research problem; the goals } \\
\text { and objectives of the project. }\end{array}$ & & & \\
\hline 1.2 & $\begin{array}{l}\text { Reviewing the Python scripting tutorials and } \\
\text { practicing Python for ArcGIS }\end{array}$ & & & \\
\hline 1.3 & Project documentation & & & 16 \\
\hline 2 & Design & & & \multirow[b]{2}{*}{10} \\
\hline 2.1 & $\begin{array}{l}\text { Make Models in ArcGIS Pro to automate tasks: } \\
\text { data cleaning }\end{array}$ & Mar. & Apr. & \\
\hline 2.2 & $\begin{array}{l}\text { ROV dive tracks Data cleaning and } \\
\text { reorganization }\end{array}$ & \multirow{4}{*}{$\begin{array}{l}\text { Apr. } \\
\text { May } \\
\text { May }\end{array}$} & \multirow{3}{*}{$\begin{array}{l}\text { May } \\
\text { May } \\
\text { Jun. }\end{array}$} & \multirow{3}{*}{$\begin{array}{l}40 \\
60 \\
30\end{array}$} \\
\hline 2.3 & System, Database design and architecture & & & \\
\hline & Project documentation & & & \\
\hline 3 & Develop/Test & & \multirow{6}{*}{$\begin{array}{l}\text { Aug. } \\
\text { Oct. } \\
\text { Oct. } \\
\text { Oct. }\end{array}$} & \multirow{6}{*}{$\begin{array}{l}40 \\
50 \\
50 \\
30\end{array}$} \\
\hline 3.1 & Creating a Geoprocessing script tool & \multirow{5}{*}{$\begin{array}{l}\text { Jul. } \\
\text { Aug. } \\
\text { Aug. } \\
\text { Oct. }\end{array}$} & & \\
\hline 3.2 & Testing the tool & & & \\
\hline 3.3 & Creating Tool Custom error reports & & & \\
\hline 3.4 & Documentation & & & \\
\hline 4 & Deployment & & & \\
\hline 4.1 & Testing the tool (Second round - piloting) & \multirow{3}{*}{$\begin{array}{l}\text { Oct. } \\
\text { Oct. } \\
\text { Oct. }\end{array}$} & \multirow{3}{*}{$\begin{array}{l}\text { Nov. } \\
\text { Nov. } \\
\text { Nov. }\end{array}$} & \multirow{3}{*}{$\begin{array}{l}25 \\
5 \\
5\end{array}$} \\
\hline 4.2 & Quality Control & & & \\
\hline 4.3 & Public presentation & & & \\
\hline 4.4 & $\begin{array}{l}\text { Developing metadata for the project's data and } \\
\text { outputs }\end{array}$ & Nov. & De & 6 \\
\hline 4.5 & $\begin{array}{l}\text { Defense and reworking (depending on the } \\
\text { defense remarks) }\end{array}$ & \multirow{2}{*}{$\begin{array}{l}\text { Dec. } \\
\text { Dec. }\end{array}$} & \multirow{2}{*}{$\begin{array}{l}\text { Dec. } \\
\text { Dec. }\end{array}$} & \multirow{2}{*}{$\begin{array}{l}8 \\
10\end{array}$} \\
\hline 4.6 & Presentation and delivering to the client & & & \\
\hline & & & Total & 420 \\
\hline
\end{tabular}




\subsection{Summary}

In this chapter, a detailed overview of the requirements of the hardware and software was provided. The design of the system was based on the agreement and needs of the client. Since the functional and non-functional requirements were essential in completing this project, they were laid out as well. A work breakdown structure showing the major tasks of the project was also provided. Next, Chapter 4 discusses the development of the database. 



\section{Chapter 4 - Database Design}

This chapter describes the data models that will be implemented for this project. The chapter will first examine the conceptual data model in an abstract way, describing all the entities and the relationships between them. Thereafter, an outline of the database structure, which was a transformation of the conceptual model into a geodatabase structure implemented for the project, will be discussed. The data sources, provided by the client, and how the data processing was done and loaded into the geodatabase will also be discussed. A summary of the entire database design chapter will be given at the end.

\subsection{Conceptual Data Model}

The conceptual data model for this project was developed through a thorough consultation with the client and it was informed by the functional database model that the organisation has in place. The conceptual model was useful in showing how the entities in mapping of the deep-sea corals and sponges relate with each other and it was important in solving the client's problems. The primary classes that needed to be defined for the model were: Survey, Event, Observation, Coverage, and Authorization, and they form tables in the database model. Others that were defined from the DSCRTP database included the Metadata, TaxonomicID and Occurrence Detail.

The Survey data describes an expedition and data collected. The transect in which records were collected and the geographical location where the events took place are described by Event data. The Observation data has the occurrence description information about coral and sponge, showing their positional data. The Occurrence, which is a table, is part of Observation data and it quantifies the various aspects of coral or sponge. The Metadata describes the provenance of the dataset. The taxonomic identification of the coral or sponge is described in the TaxonomicID class. Finally, the Occurrence Detail quantifies the various aspects of the coral or sponge (e.g., number or weight) and other biological details (e.g., condition and biological associates). The conceptual model of this project is illustrated in Figure 4-1.

While on a research Cruise to survey deep-sea corals, sponges and fish habitats, the ROV is attached to the Okeanos Ship Explorer using a umbilical cord which transmits power and communication signals while observing the ocean bottom. When the ROV is descended into the ocean, observations are made and the locations with positive recording of corals and sponges as seen from the live videos, the location is saved with latitude and longitude and the other Observation Data is recorded. When the ROV is taken back to the ship, that is counted as one dive. Typically, one dive takes very long to complete. The process of surveying deep-sea corals and sponges using ROVs can take up to eight hours to survey a stretch of one kilometer.

A dive, observations done between one descend and an ascend of ROV, must contain survey data which describes the event data. The event data contains the observation data where information about coral and sponge is recorded. The observation data may contain an image of the individual observation point. 


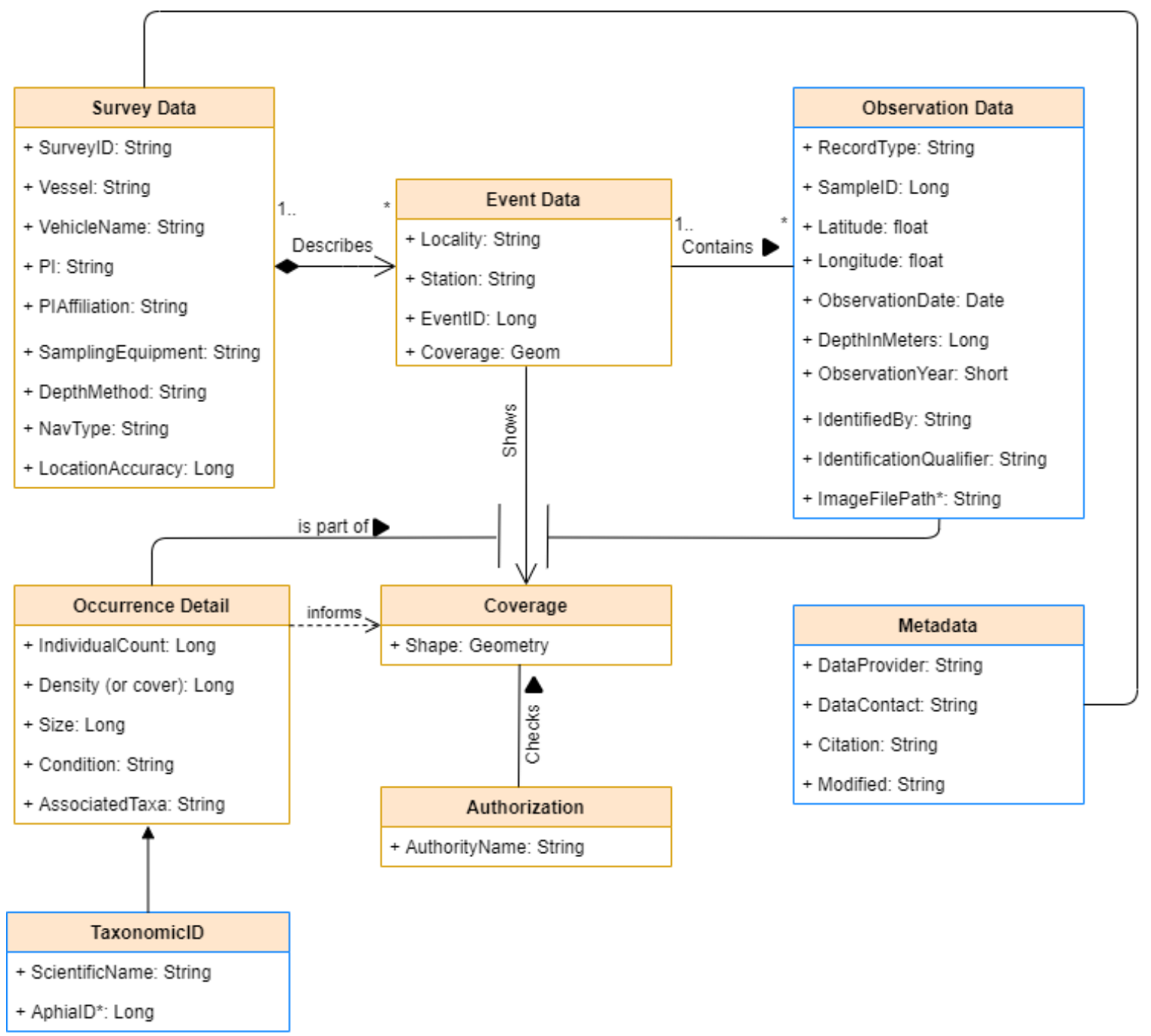

Figure 4-1: Conceptual Data Model

\subsection{Logical Data Model}

Before this database was developed, several aspects had to be considered. First, the feature(s) to be included in the database were to be thought about. As an illustration, in ArcGIS, modelling is either done as points, lines or polygons and therefore, realizations must have these characteristics assigned to them. Secondly, looking at how the data creation would be done was important. Finally, the usage of the data whereby, by knowing the queries (such as the density of corals and sponges found in a defined surveyed area of the deep-sea) that can be performed would help in shaping the database. Therefore, with the above information in mind, a logical model was a more detailed structure of the implementation of the database schema. The logical model implemented for this project considered the best way of storing the collected Remotely Operated Vehicles (ROVs) observation data for deep-sea corals and sponges. The design of our database started with a well thought out process about the dataset, which was to be housed in database, the way the data was to be edited and maintained over time. In this database, there are two feature classes: the ROV dive tracks and the feature class 
representing the ROV mapped areas representing the input and output respectively. However, as a way of making sure that the scope of the project remained specific as outlined at the beginning of the project, not all entities discussed in the conceptual model implemented in the geodatabase. Figure 4-2 shows how the logical model was implemented in the geodatabase.

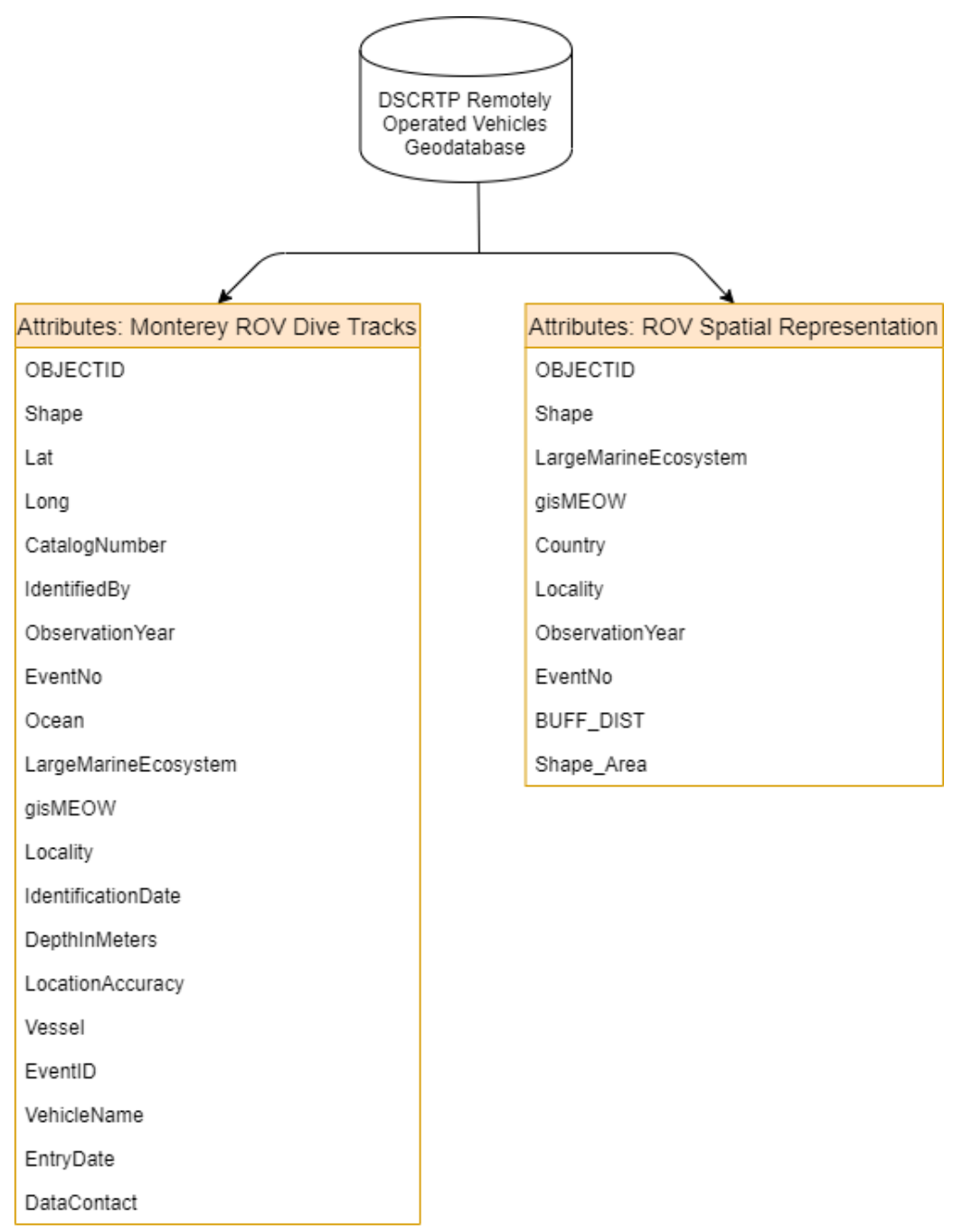

Figure 4-2: Logical database schema

\subsection{Data Sources}

In order to accomplish the needs of the client, the data was playing a very significant role in the implementation of the overall goal and objective of this project. The data and metadata that was used was readily available from the client. Figure 4-3 shows a few rows of the of the ROV data. 


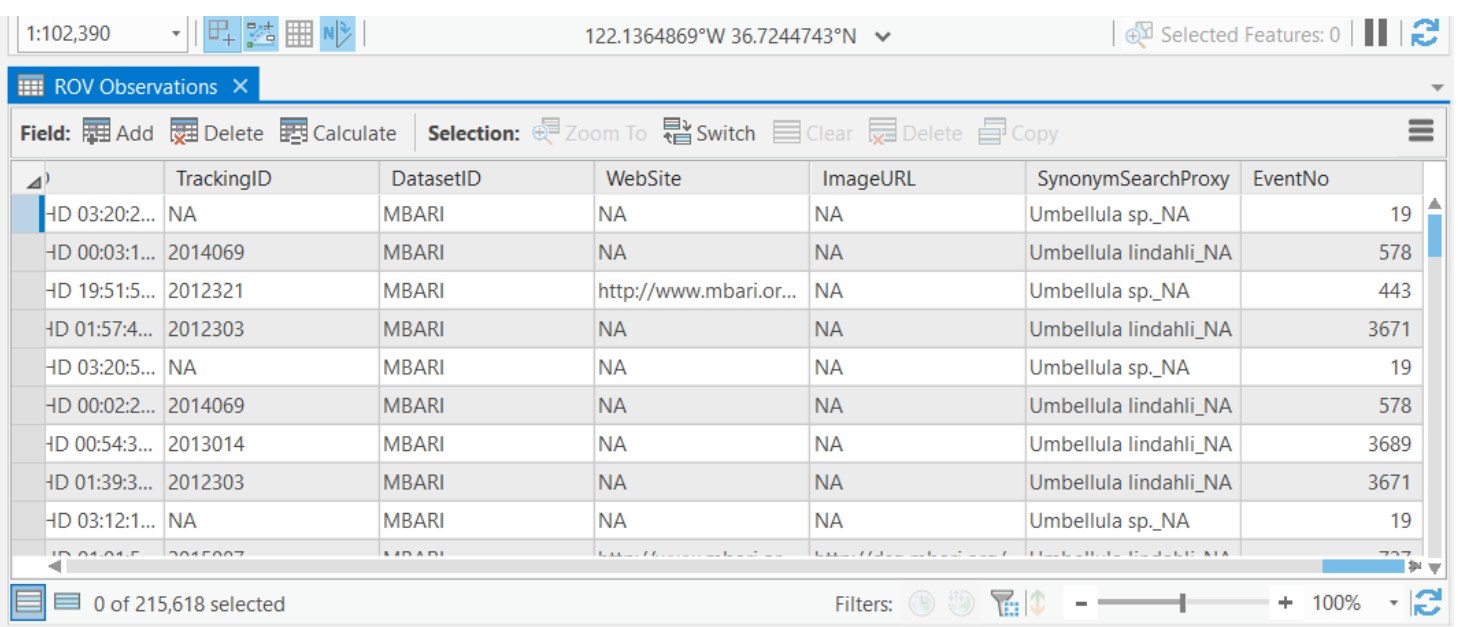

Figure 4-3: The Attribute rows of Remotely Operated Vehicles Dive observations data

The dataset (named Monterey_Deep_Sea_Corals_ROV.csv) was supplied in the format of a comma separated value (CSV) and it contained 217,037 dive track point observations. This was the only dataset that was used for this project. These dive track observations were collected using three types of ROVs namely: ROV Doc Ricketts, Ventana ROV and Tiburon ROV. Figure 4-4 shows the images of the three ROVs. The location accuracy of the dive tracks data was recorded as 100 meters. This data had a metadata file as well (a csv file named 20190314-

0_DSCRTP_National_Database_Dictionary). This metadata was crucial in helping to establish what the fields in the dataset were and how to interpret them. 


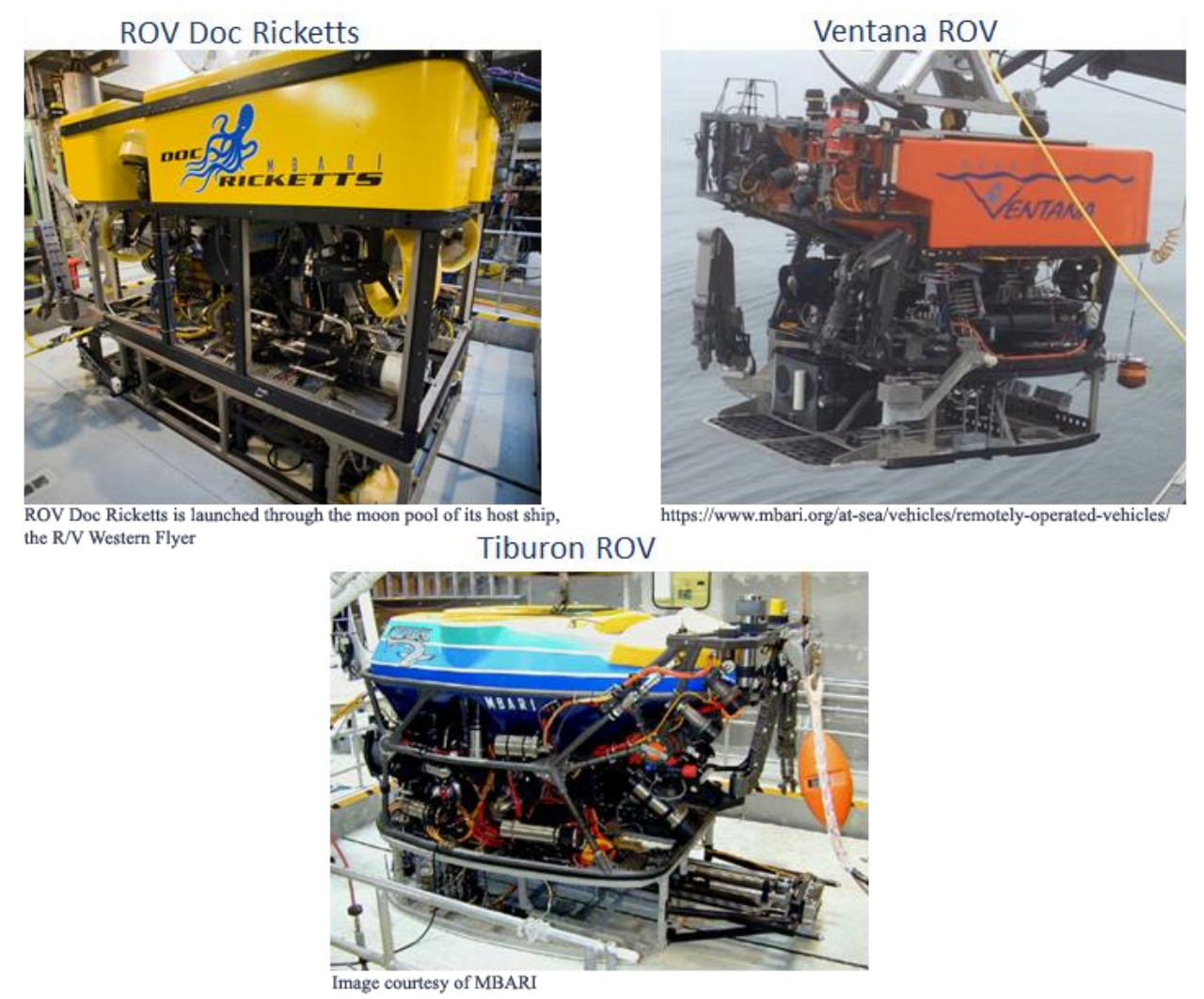

Figure 4-4: The ROVs used to collect the dive tracks observations

\subsection{Data Scrubbing and Loading}

The project's ROVs dive tracks data used for analysis was provided by the client in Excel spreadsheet format. Using ArcGIS Pro version 2.4.1, a geodatabase was created to help in storing and organizing the data. Before loading of the data into the geodatabase, an Event feature class schema was developed. The longitude and latitude columns were stored as the first columns in the Excel file to ensure they were drawn properly on the map. Upon converting the data into a feature class, it was then loaded into the geodatabase and projected to WGS 1984 coordinate system.

\subsection{Summary}

In this chapter, the conceptual data model, logical data model, sources of the data, and the prior data cleaning that were performed before analysis was discussed. The data models: conceptual and logical, were used to describe the classes and the relationship they had as well as the geodatabase structure. The ROV dive tracks data that was used for analysis came from a reliable source and it also included a rich and helpful metadata. 


\section{Chapter 5 - Implementation}

As discussed earlier, this project was aimed at spatially representing the remotely operated vehicles (ROV) dive tracks to help in showing the areas that have been covered in the previous expedition surveys. Therefore, after the compilation of a database in the previous chapter, this chapter discusses the implementation of the tools that were created for data reorganization and an analysis tool to help achieve the main aim of the project.

\subsection{Geoprocessing services}

This project implemented two geoprocessing services. A model builder tool was built for reorganizing the data and a custom tool was built to produce the areas surveyed in the deep-sea. The tool was built using the modelbuilder in ArcGIS Pro version 2.4.1 Toolbox.

\subsubsection{Add Event Number Model Tool}

The most crucial part of this project involved the organization of the data used for analysis. The initial data that was supplied by the client needed to be pre-processed before analysis could be performed. The issues that the dataset had included: the wrong data type, observations that did not represent deep-sea corals and sponges (those that were taken in depths not more than 50 meters) were removed and the observations that missed to have an event identity. Therefore, to solve the mentioned data issues, a modelbuilder tool in ArcGIS Pro had to be built to automate the creation and calculation of a new Event Number field in order to have the required data type, integer, of events identities.

The Deep-Sea Corals and Research Technology Program (DSCRTP) provided the Remotely Operated Vehicles (ROVs) data in the comma separated format (csv), which was collected between 1989 and 2015. The data contained a total of 217,037 ROV observations of corals and sponges in the Monterey Bay, Los Angeles, Gulf of California and Hawaii sections of the deep-sea.

After the acquisition of the data, the first process involved loading the data into ArcGIS Pro 2.4.1 using the XY Table to Point tool which is available in the Toolbox to create a feature class named Monterey ROVs. The XY Table To Point tool helps to create a new point feature class based on the $\mathrm{x}$ (longitude), $\mathrm{y}$ (latitude), and $\mathrm{z}$ (depth/height) coordinates from the table being loaded. For this tool to function, the standard delimiter for tabular text files allowed should have a .csv or .txt comma extension or .tab extension. The World Geodetic System (WGS) 1984, which is the XY Table To Point tool default coordinate system, was used for the Monterey ROVs output feature class. The feature class was later re-projected to North America Albers Equal Area Conic and stored in a

file geodatabase created for this project in ArcGIS Pro. North America Albers Equal Area Conic projection was suitable for the study area since it maintains accurate area measurement and maintains the scale along the two standard parallels. However, it should be noted that this projection distorts shape farther away from the two standard parallels. Since this feature class had many column fields which were not used for the analysis, their visibility was hidden in the table to allow quick search and editing of the fields that 
were used for analysis. Hiding the visibility of a column in a table is important since the field is no longer visible on other dialog boxes, such as the Pop-up window when you identify a feature, and the Attributes pane for the selected features you want to edit.

The Add Event Number tool consisted of four parameters: input ROVs Table, New Field Name, Field Type, and Field Alias. These fields were specified during the creation of the tool. This tool was used to create an integer field named EventNo with Event Number as the alias. The values of the field were then calculated based on EventID field in the ROVs Observation data. Changing the data type of the EventID from text to an integer was important since it helped in identifying the observations that had been recorded with more than one event identity number. Figure 5-1 shows some of the fields that were not used for analysis while Figure 5-2 shows an overview of the tool.

\begin{tabular}{|l|r|}
\hline \hline EventID & EventNo \\
\hline 479,480 & $<$ Null $>$ \\
\hline 479,480 & $<$ Null $>$ \\
\hline 479,480 & $<$ Null $>$ \\
\hline 479,480 & $<$ Null $>$ \\
\hline 479,480 & $<$ Null $>$ \\
\hline 700,701 & $<$ Null $>$ \\
\hline 1506 15n7 & -Null> \\
\hline
\end{tabular}

Figure 5-1: The Null observations that were not used to produce areas surveyed in the deep-sea

Figure 5-2: Add Event Number Tool User Interface

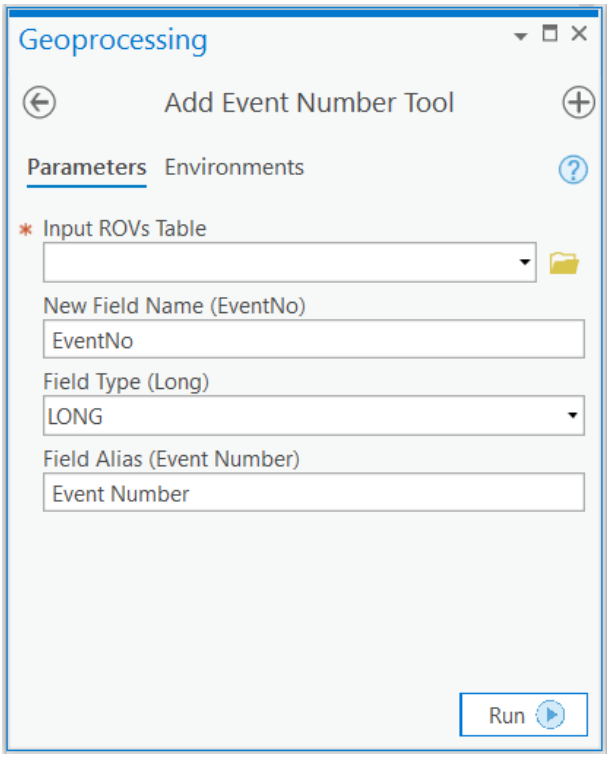

\subsection{Options for ROV Surveyed Areas Representation}

Before this project settled on implementing the ROV Survey Area Generator Tool, several options: using the Points to Line, Aggregating Points, and Buffer approaches, 
were tried. The discussion below assesses the approaches and why each of them was considered not a viable option for representing the ROVs surveyed areas in the deep-sea.

\subsubsection{The Points To Line Approach}

This tool allows the point features to be converted into lines based on a unique field in the input feature table. Figure 5-3 shows the expected output of the Points To Line tool in ArcGIS Pro. The aim of this approach was to connect all the unique dive points in a line and thereafter, buffer the line based on the view capability of the ROVs used in mapping the deep-sea corals and sponges. This approach was not effective since the user would not have the chance of specifying more than one field to draw lines uniquely. As a result, when one field, from the ROVs data, was used to uniquely group the dive tracks, lines drew all over the space with some points coming from the same points more than once as shown in Figure 5-5.

The tool was producing lines drawing all over the ocean space of the study area since it was only limited to specifying one unique field to produce the result. Also, the data didn't have a unique sorting field. Therefore, to achieve in having each point observation in the same dive represented as a unique line, more than one field (Country, EventNo, gisMEOW, Locality, Observation Year and LargeMarineEcosystem) had to be specified.

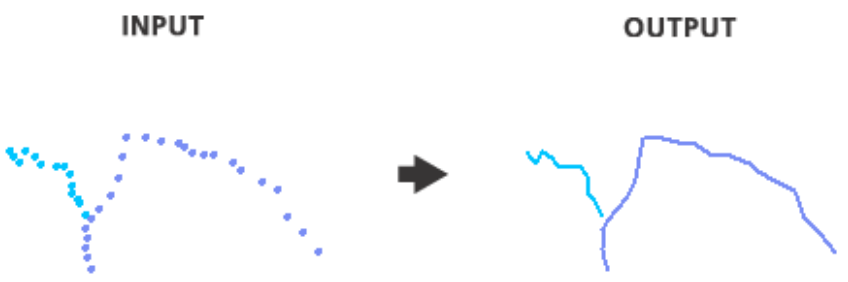

Figure 5-3: An illustration of How Points To Line tool is supposed to work. (Source: Esri Points To Line tool documentation)

Figure 5-4 shows the Points to Line tool in ArcGIS Pro. The Line Field parameter of this tool allows a unique value to be selected and which each feature in the output will be based on. This option only allows one field to be chosen to uniquely produce the output. The Sort Field parameter also accepts only one field and it's important in specifying the order that the points should draw, either by the way they appear or choose a desired approach. 


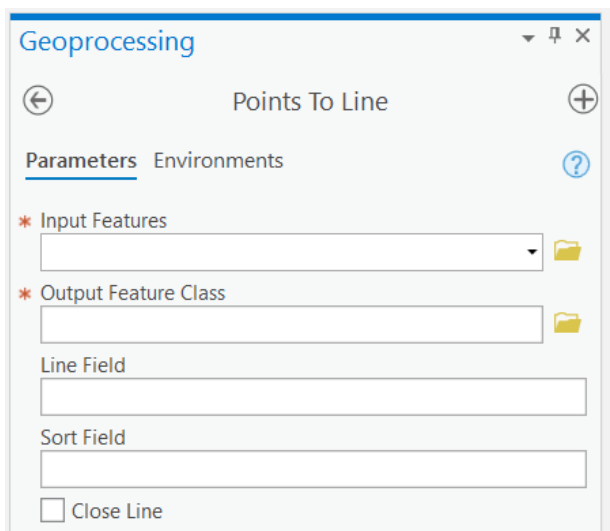

Figure 5-4: The Points To Line tool in ArcGIS Pro showing the parameters that the tool accepts

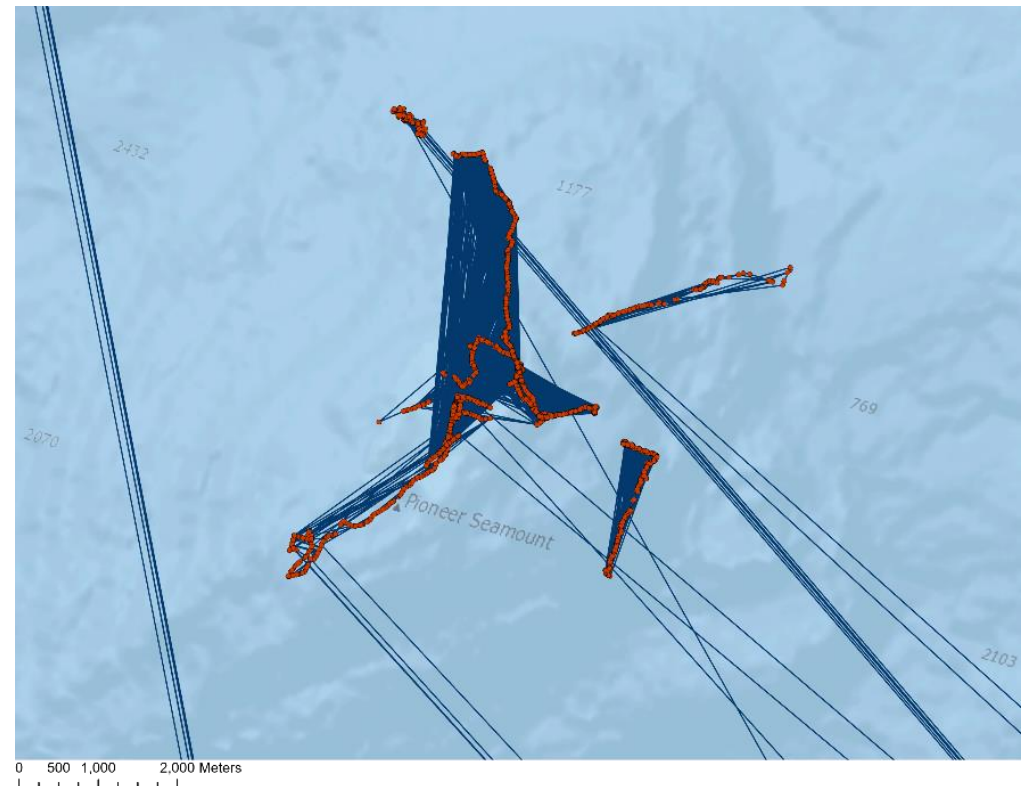

Figure 5-5: Example of the output of Points To Line tool

\subsubsection{The Aggregating Points Approach}

This approach aimed at producing an output with a count of points and all the locations where dive observations were occurring. This approach was focused on aggregating the projected ROV observation points into polygon features or bins. In this option, a square bin of $500 \mathrm{~m}$ by $500 \mathrm{~m}$ size that was generated when the analysis specified the areas into which the ROV observation points were to be aggregated. Figure 5-6 shows the result of the tool which is a polygon layer that is returned only where points occur. Based on the feedback given by the client, this was not an ideal method of representing the surveyed areas. In a square of $500 \mathrm{~m}$ by $500 \mathrm{~m}$, a polygon would be drawn even if only one observation was made in that location thus this would end up being counted as a surveyed area. Therefore, this would mean that the possible covered areas was being overrepresented. 


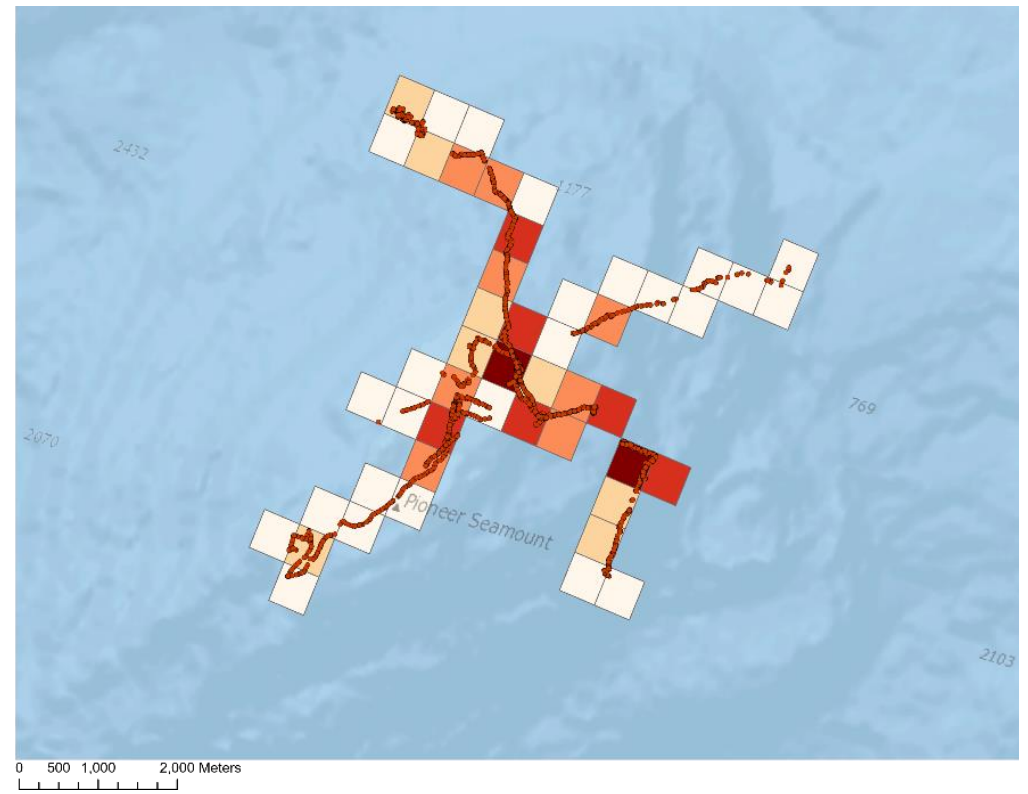

Figure 5-6: Example of the output of Aggregate Point tool from GeoAnalytics Server toolbox with each square measuring 500 meters by 500 meters

\subsubsection{The Buffer Approach}

A buffer routine traverses each point of the input feature's vertices and creates buffer offsets. Figure 5-7 illustrates how buffer works in ArcGIS Pro, where two basic methods of buffering have been implemented. These methods are Euclidean and geodesic buffering. In Euclidean buffers, the distance is measured in two-dimensional Cartesian plane where straight lines are calculated between two points on a flat surface. Euclidean distances work well when analyzing distances in features projected in a single coordinate system such as a single UTM zone. On the other hand, Geodesic buffers consider the shape of the earth, a geoid, and distance calculation between two points is based on a curved surface (the geoid).

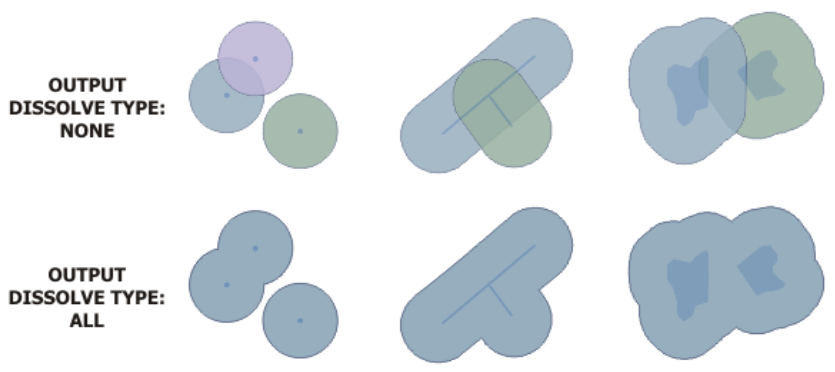

Figure 5-7: An illustration of how Buffer tool is supposed to work. (Source: Esri Buffer tool documentation)

With the above information in mind, it was difficult to decide on the viewshed distance that was to be applied to the ROV input features. In the example of buffer output shown in Figure 5-8, a geodesic method was selected with a buffering of 100 meters (the 
value of location error recorded in the input dataset). With the six unique fields specified for the buffer tool and the viewshed distance (buffer distance) specified, this method was not ideal since for example, with a distance of 100 meters, even if observations were in the same track and they were spaced more than a 100 meters, that would leave a gap. This gap would be taken as a region which had not been surveyed whereas an ROV could have passed through that section.

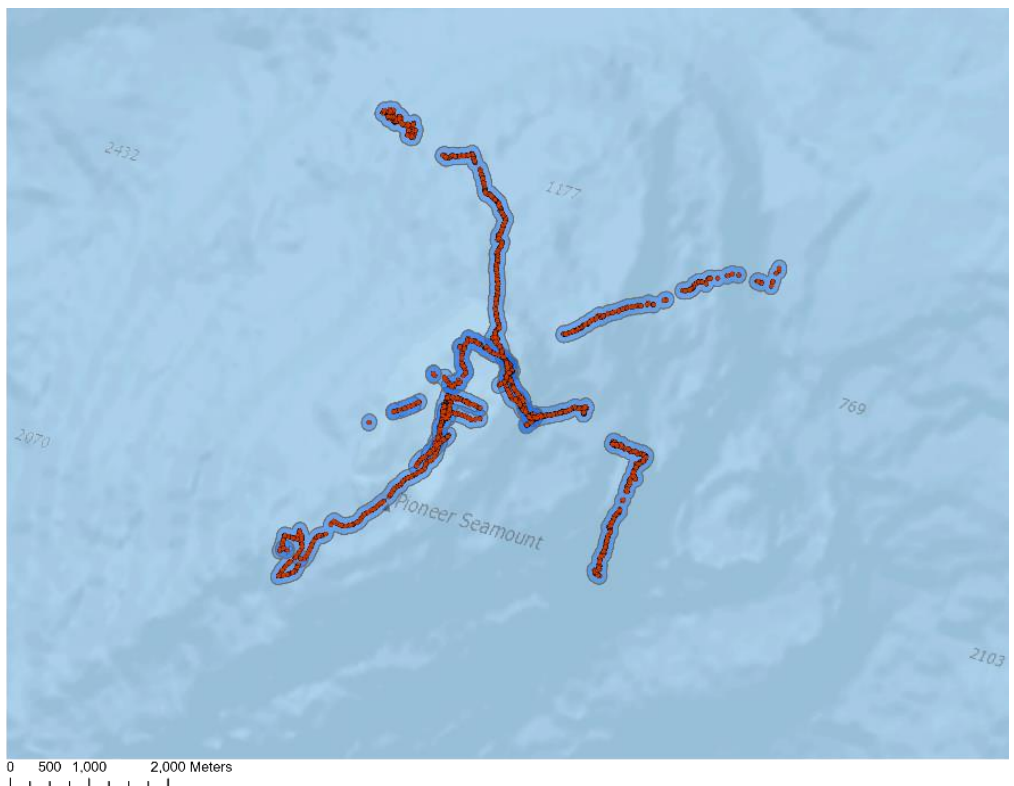

Figure 5-8: Example of Buffer tool output produced using a buffer of 100 meters

\subsection{Realization of the ROV Survey Area Generator Tool}

Figure 5-9 shows the modelbuilder tool created to help in implementing the goal of the project: to show the areas that surveys using ROVs had taken place. The user had to specify the input features as the ROV dataset and then save the output features in a local directory. By default, the geometry type was chosen as Rectangle by Area to give a rectangle of the smallest area enclosing the input features. The other very critical entry of the tool was the decision on grouping options. Since the dive events comprised of groupings occurring in different locations, as well as records of different years in time, List option was chosen as the grouping options. This would give a user of the tool the capability of specifying the fields from the input dataset to be used in grouping the dives. As shown in Figure 5-10, the modelbuilder tool also allows the user to define the ROV viewshed (buffering distance), specify the buffering method as well as the fields to dissolve thus removing any buffer overlaps. 


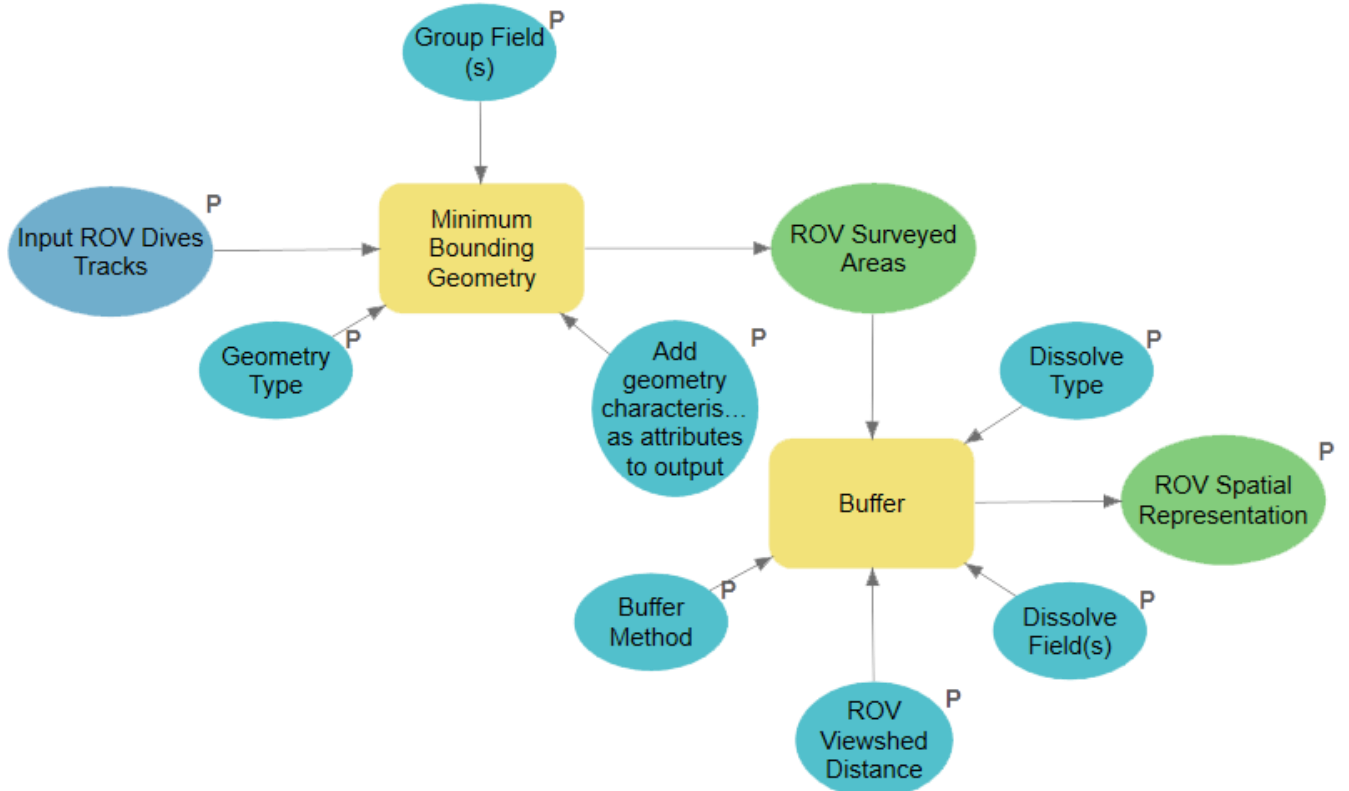

Figure 5-9: ROV Survey Area Generator ModelBuilder 


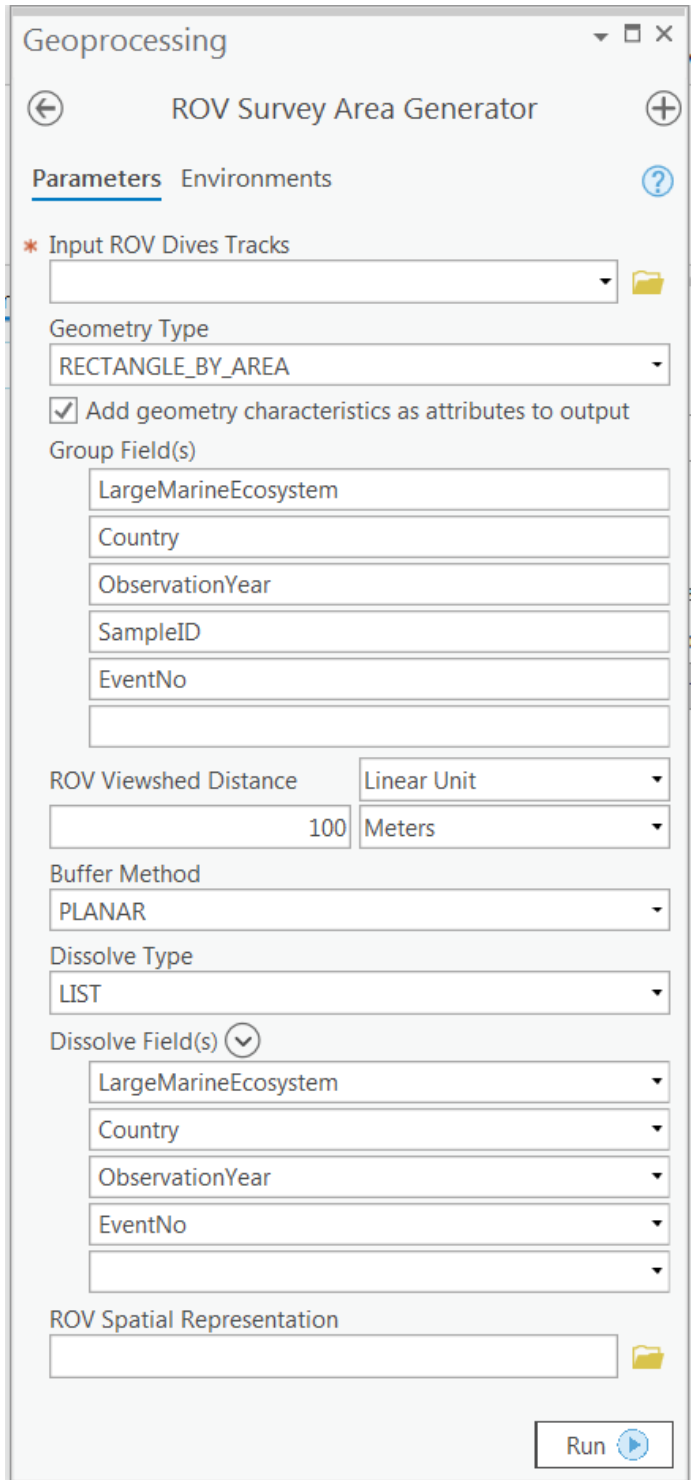

\section{Figure 5-10: The ROV Survey Area Generator Tool}

This project uses five distinct fields found in the dataset: LargeMarineEcosystem, Country, ObservationYear, SampleID and EventNo. However, it is important to mention that the order of listing these key fields in the ROV Survey Are Generator Tool is important in ensuring that the fields in the attribute table are consistent and represent each mapped field. Figure 5-11 shows the attribute table of the product generated by the ROV Survey Area Generator Tool. 


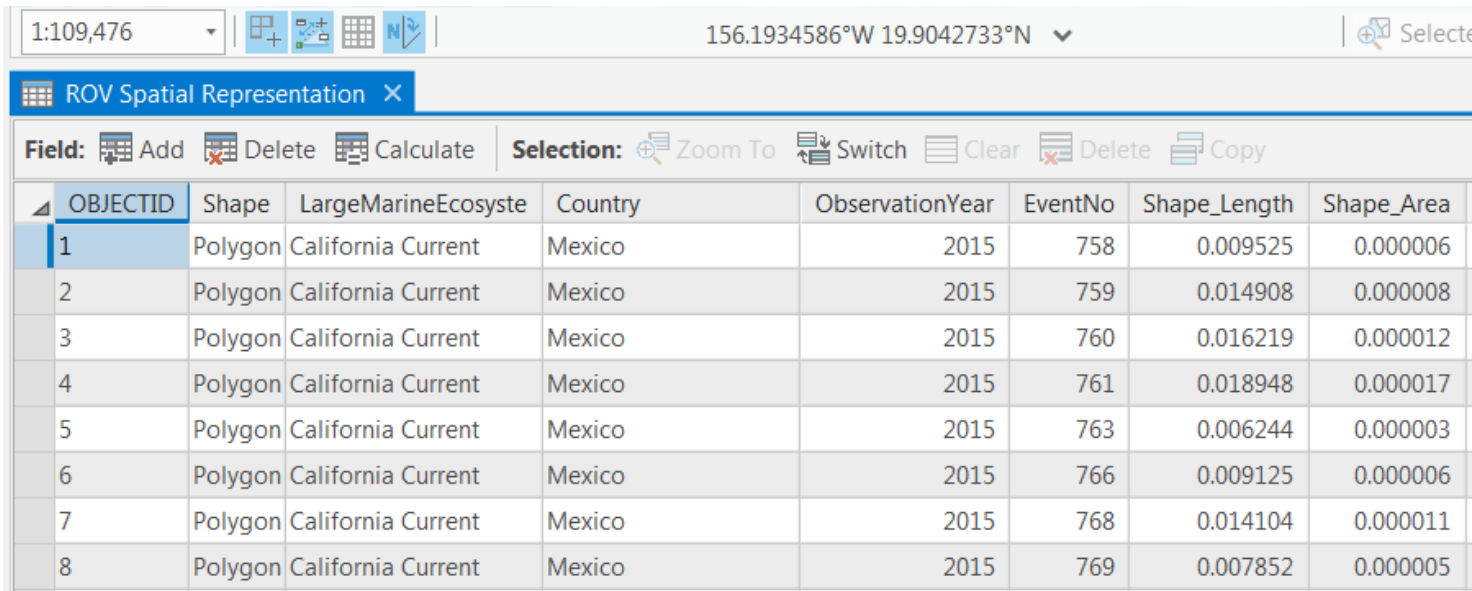

Figure 5-11: A view of the attribute table for ROV spatial representation

Table 6 lists the five fields and their descriptions. For the dissolve option of the tool, four fields, LargeMarineEcosystem, Country, ObservationYear, and EventNo, were chosen in order to eliminate the buffer overlaps created after running the tool.

Table 4. The fields used to group the ROV data points

\begin{tabular}{|l|l|}
\hline Field Name & Description \\
\hline LargeMarineEcosystem & Large Marine Ecosystem using set designations \\
\hline Country & $\begin{array}{l}\text { Country in whose Exclusive Economic Zone the } \\
\text { observation or sample was collected, based on } \\
\text { Exclusive Economic Zones Boundaries (EEZ) }\end{array}$ \\
\hline ObservationYear & The year that the observation was made \\
\hline SampleID & $\begin{array}{l}\text { The primary identification label or code of the } \\
\text { specimen or catch record exactly as reported by the } \\
\text { Data Provider. }\end{array}$ \\
\hline EventNo & $\begin{array}{l}\text { ID number of the survey event (e.g., dive number, } \\
\text { transect, or trawl haul number) on which the } \\
\text { sample was made. }\end{array}$ \\
\hline
\end{tabular}

The grouping option was so critical, since the dive tracks had been collected in different locations of deep-sea and at different years, and separating the dives based on the mentioned fields would allow a more precise representation. Figure 5-11 shows an example of a dive 80 collected in the years 1989 and 2009, which is a possible occurrence as described by the client. This clearly shows why grouping was necessary. 


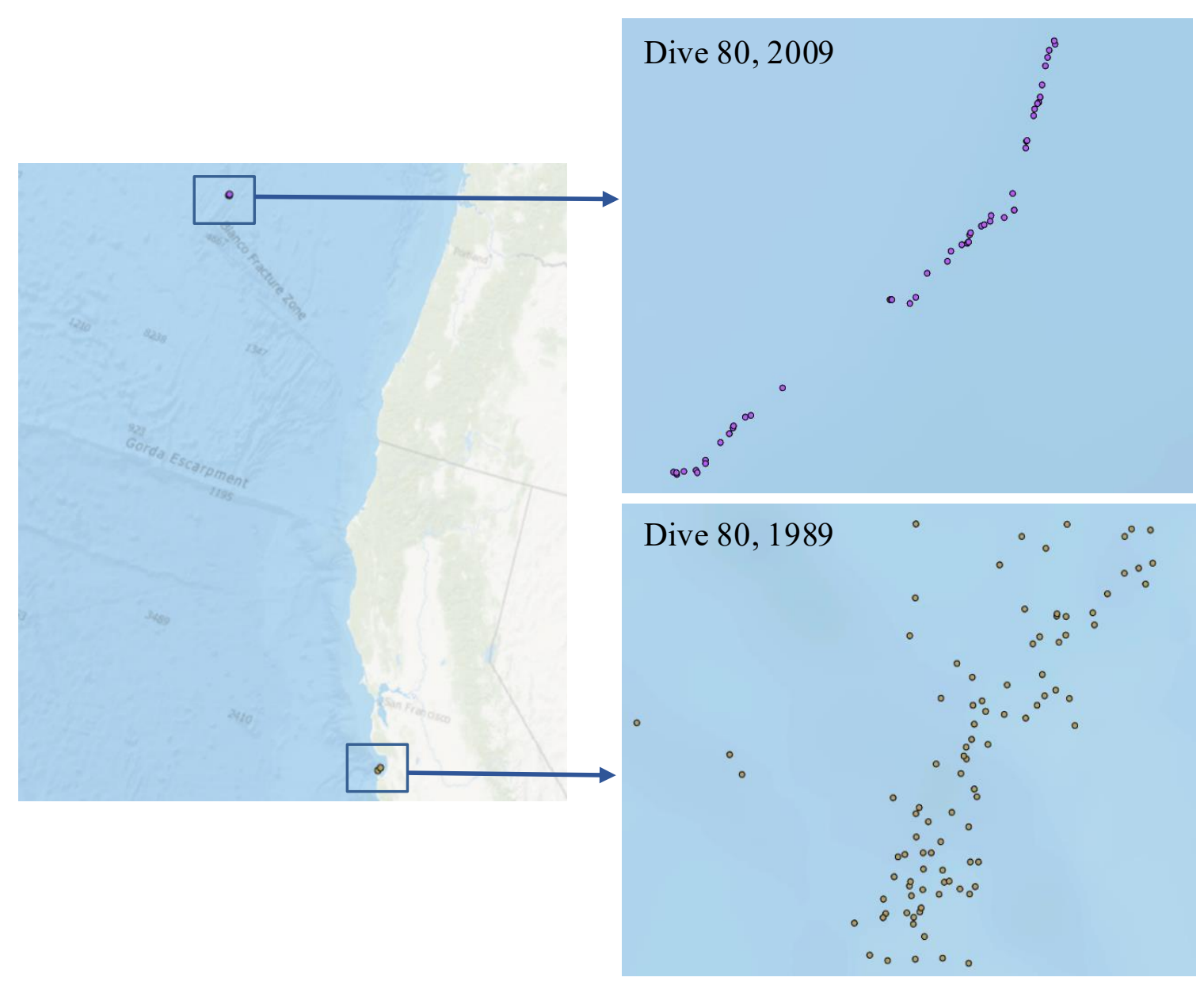

Figure 5-12: Dive 80 showing dives with the same EventNo but recorded in different years. This shows why grouping was vital to avoid such issues.

\subsubsection{Analysis and Output of the ROV Survey Area Generator Tool}

The ROVs Survey Generator Tool requires the user to first specify the input feature which is the ROV dive track observations. After the input is specified, the user must choose the geometry type as a rectangle by area and ensure that the option of adding geometry to the output is ticked. This option ensures that geometric attributes are added to the output features. The next key entries of the tool are the groupings fields where the user must list the unique fields used to group the observations. Finally, the user must also define the output feature, name and location in the local directory.

Once the user had specified all the required inputs and output of the tool, the analysis was performed to produce the areas surveyed in the deep-sea using ROVs. In Monterey Bay, a lot of observations had been conducted thus resulting in many overlaps of polygons showing observation footprints. Figure 5-12 shows the overlaps of areas covered by the ROV surveys in Monterey. Figure 5-13 shows a superimposition of the ROV dive tracks with the coverage areas. 


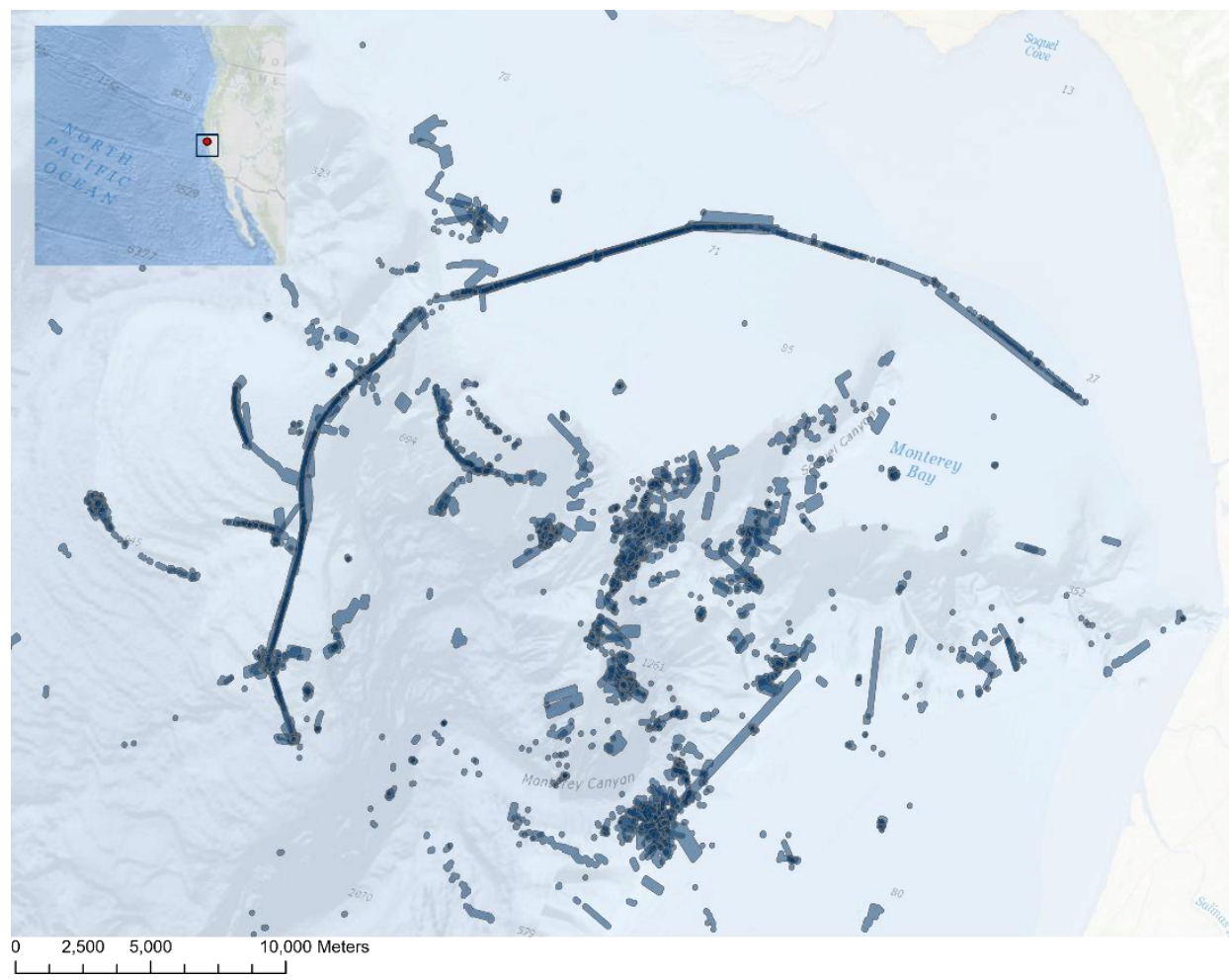

Figure 5-12: A section of ROV coverages in Monterey Bay

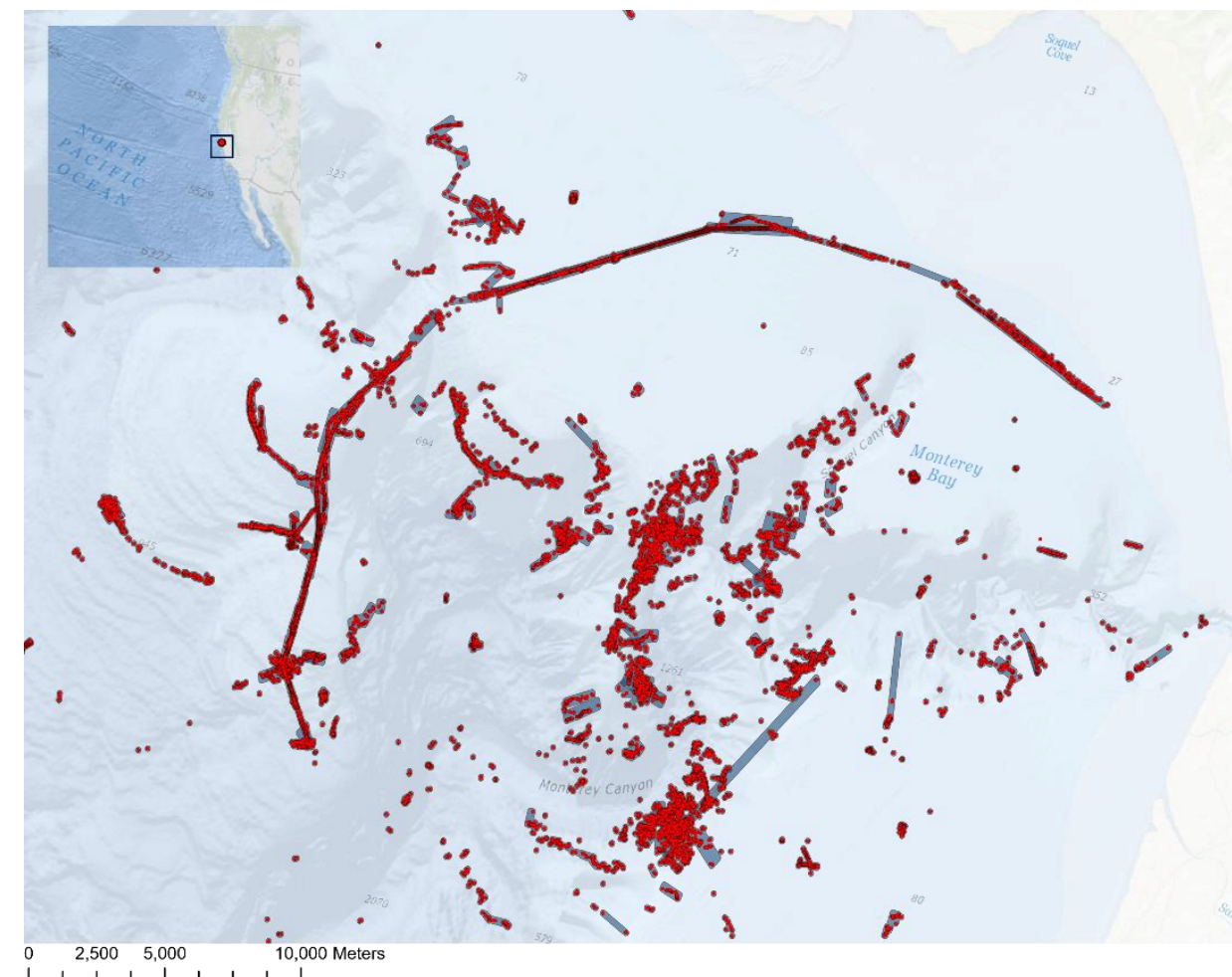

Figure 5-13: A section of ROV coverages in Monterey Bay superimposed with ROV dive track observations 
As shown in Figure 5-14, in the Hawaiian area of the deep-sea, the areas that ROV observations had been conducted were produced.

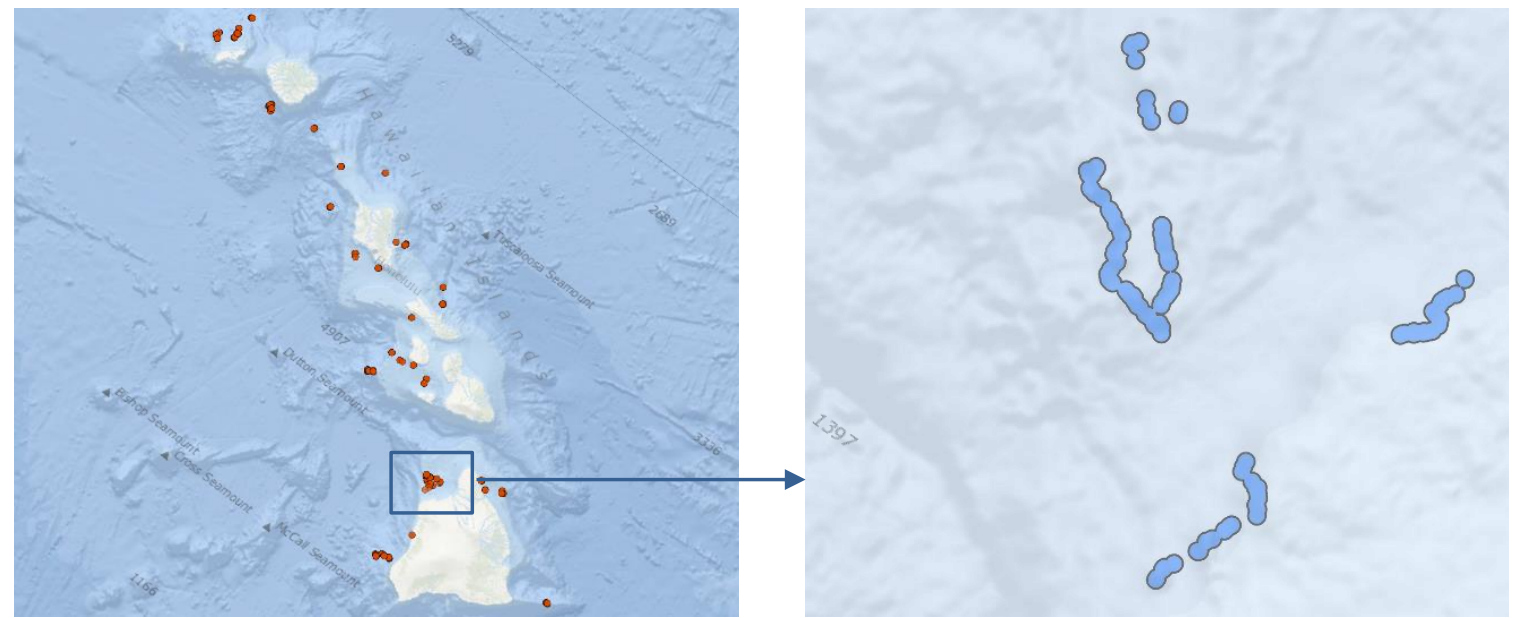

Figure 5-14: ROV representation of a dive near Hawaii

\subsection{Summary}

Since prior data reorganization and cleaning was a crucial step before any further analysis, a tool which was created in ArcGIS Pro version 2.4.1 for automating this process has been discussed in detail in this chapter. Also, in the chapter, the usage of the custom tool created for producing the areas that have been surveyed by the ROVs was extensively discussed. 


\section{Chapter 6 - Results and Analysis}

Following the implementation phase discussed in Chapter 5, this chapter will discuss the results and analysis of the project. First, the chapter will start by discussing why this project was useful to the client by highlighting the major use cases of the deliverable. Thereafter, the chapter will discuss possible methods that could be used to represent the areas where a survey using an ROV had already been conducted. This discussion will also include the reason why none of the methods was a potential approach in helping to achieve the goal of the project. The evaluation done in this section does not reflect National Oceanic and Atmospheric Administration (NOAA) or Deep-sea Corals and Research Technology Program (DSCRTP), but rather, it's a reflection of the tool used for analysis.

\subsection{Use Cases}

In the deep-seas, corals and sponges are very crucial in forming rich biogenic habitats. Most of these corals and sponges take up to hundreds of years to reach maturity and therefore, once destroyed, it means that species such as triggerfishes, wrasses, damsel fishes, crabs, snails, sea stars, butterfly fishes, shrimps, parrot fishes, sea urchins among others, which depend on these corals and sponges for food, shelter or breeding zones are greatly affected. By helping to show the areas that have been covered by ROVs during a search for corals and sponges in the deep-sea, this project is very critical in the decisionmaking process. This includes the decision on issuance of permits to exploit parts of the deep-sea based on whether an area has been explored or not and the density of corals and sponges in such areas. The possible applications of the project's deliverable are discussed in the following section.

\subsubsection{Deep-sea Mining Activities}

Deep-sea mining is a major threat to marine life and especially to the deep-sea corals and sponges. Therefore, companies that are involved in extensive mining activities in the deep-sea, need to ensure that their target area of exploitation has no or has minimum density of species such as corals and sponges, before undertaking any activity. In the United States, the regional fisheries and management council has a mandate to decide on who, where, when, and how to exploit a certain section of the deep-sea. Following this, if a deep-sea mining company is interested in exploiting an area of the seafloor for its expected high mineral potential, a reference of whether that area has been explored for deep-sea corals and sponges would be the first thing to check upon, when looking at other characteristics such as the density of corals and sponges before issuing a permit of exploitation. Figure 6.1 shows some of the effects of deep-sea mining to species.

The means the precision of the methods used to determine if an area has been covered or not are very key. As demonstrated in Figure 6.2, different methods produce different coverage areas. As an example, if the Aggregate approach was to be used as a reference of whether an area has been mapped, the areas which have not been covered during a survey would be covered by bins. This would lead to making misinformed decisions on how the deep-sea is to be exploited. 


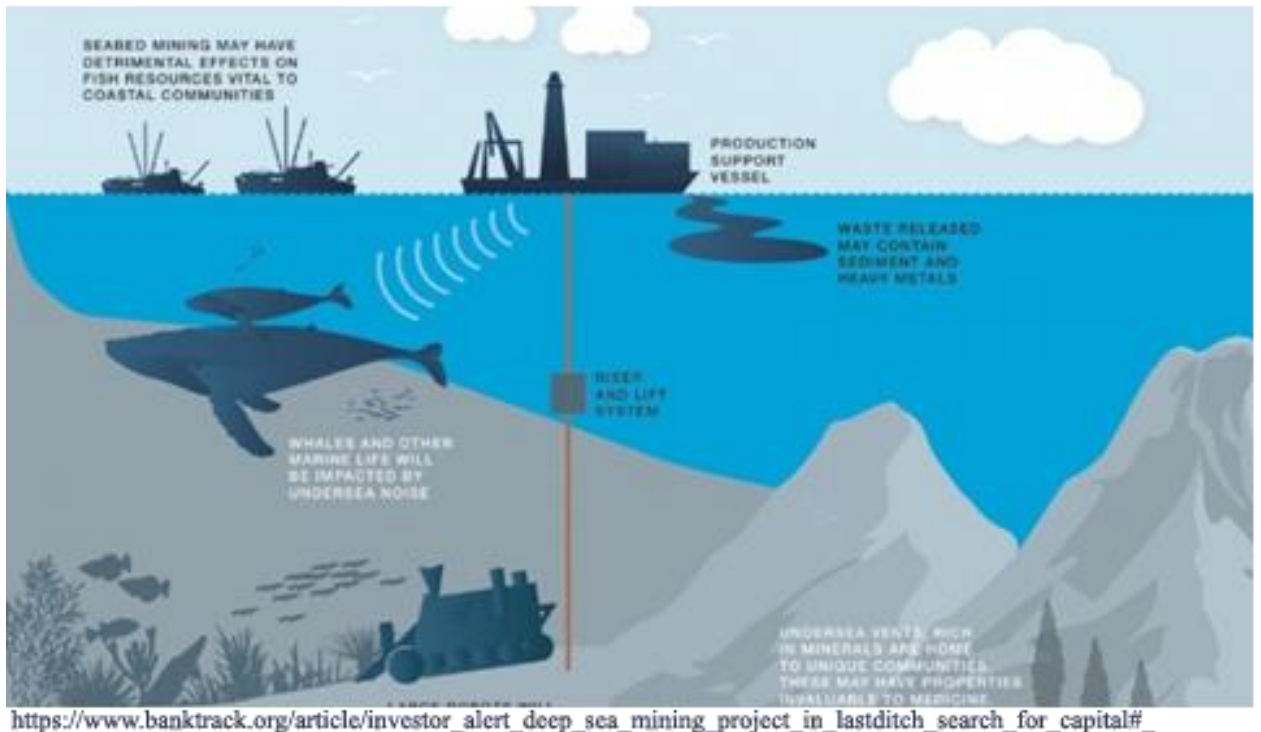

Figure 6-1: Schematics of deep-sea mining process

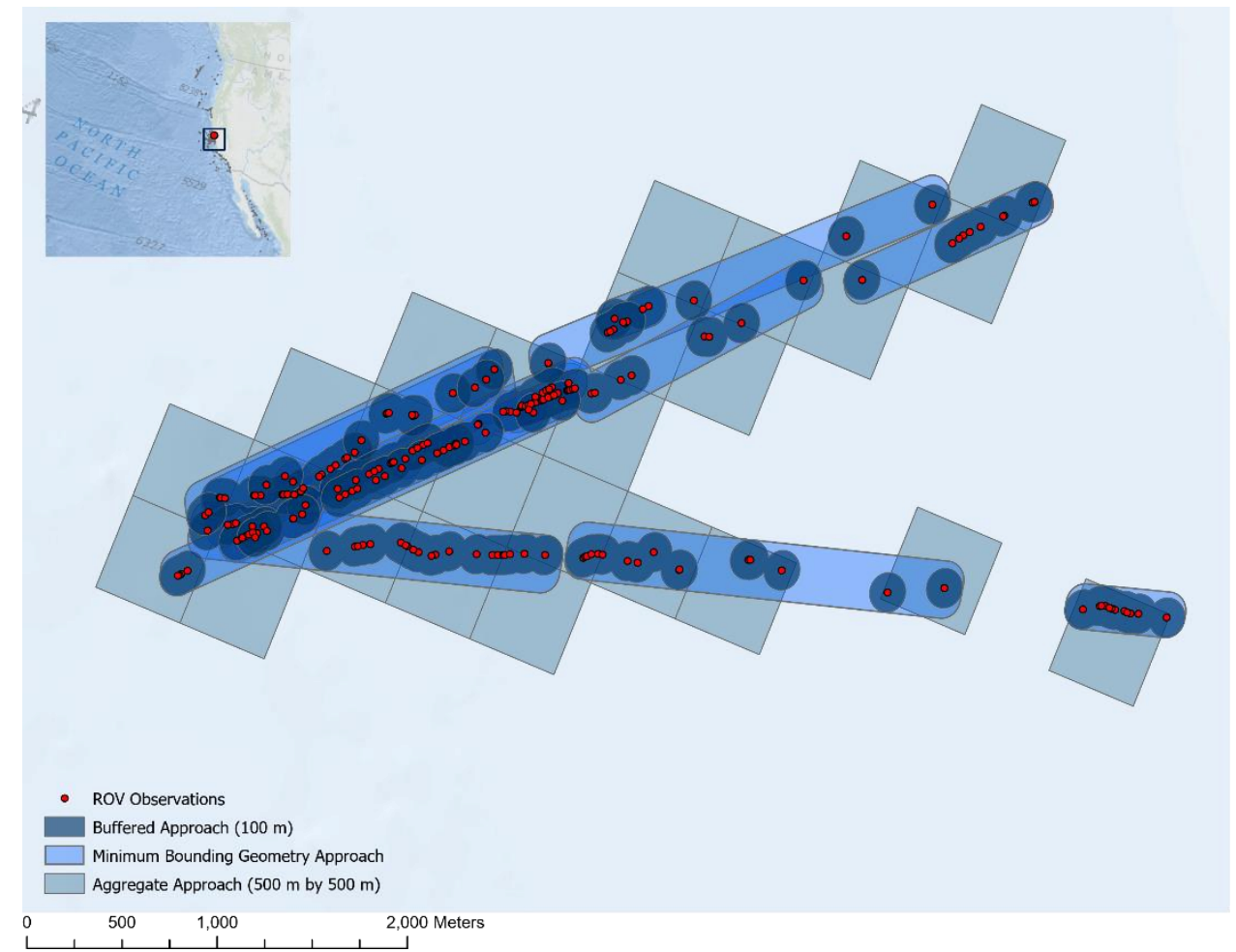

Figure 6-2: A comparison of coverage areas in a section Monterey Bay showing the usage of the Aggregate Points, Minimum Bounding Geometry and Buffer approaches

\subsubsection{Deep-sea Bottom Trawling Operations}

Bottom contact trawling is a method of deep-sea fishing where a net with heavy weights is dragged on the seafloor thus scooping up everything that falls on the trap's way. This 
method can be very destructive to species when done on benthic zones, where a rich diversity of species such as fish, corals and sponges occur. In such a scenario, following the powers given to the Fisheries and Management Council by the Magnuson Stevens Act (MSA), a decision must be made prior to administering exploitation permits. Looking at whether an area has or has not been surveyed for deep-sea corals and sponges would be the first thing that the council would do before opening an area for bottom-contact traps. Therefore, with this project's deliverable being coverage areas, the fisheries and management council would be able to establish which areas should be permitted and those areas that should stay closed to fishing due to their highly sensitive biodiversity in the deep-sea. Figure 6-3 shows a bottom trawling method in the deep-sea and how it can be detrimental to corals and sponges.

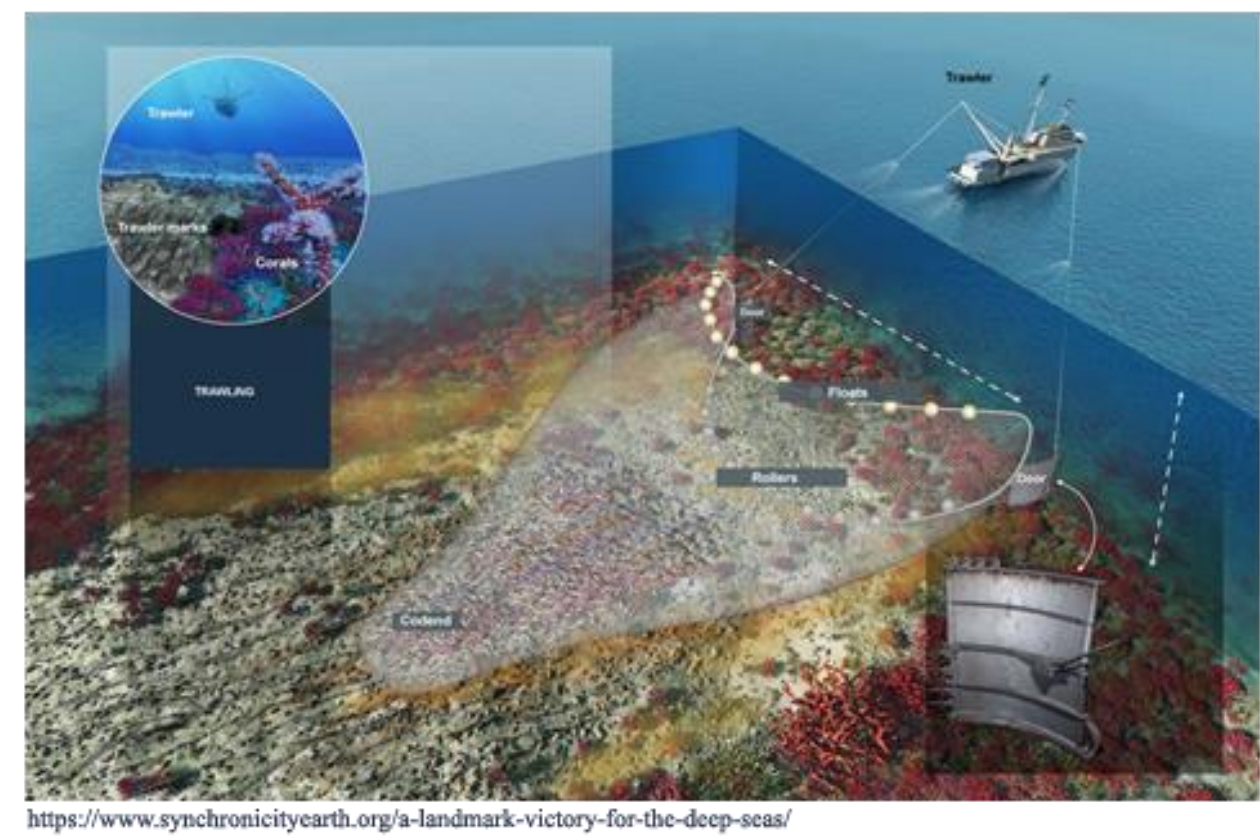

Figure 6-3: Bottom trawling

\subsubsection{Other Applications of the Project}

Relative to the previous discussion, other key sectors that would highly call for establishing whether an area has been surveyed or not before administering a permit would include deep-sea infrastructure like cables, and open aquaculture. The latter is a major driver of acidification in the deep-sea and this would result in the ultimate death of corals and sponges. Before any of these operations are undertaken, an area must be surveyed so that the information on density and numbers of species such as fish, corals and sponges can be established.

\subsection{The Analysis and Results of the ROV Survey Area Generator Tool}

As discussed earlier in Chapter 5, the ROV Survey Area Generator tool was taking the dives as the input features. This tool was creating a feature class containing polygons which represented a specified minimum bounding geometry, enclosing each dive group 
input feature. The corals and sponges observations, which served as the input features for the tool, had been recorded between the years 1989 and 2015. The output of this tool was a polygon feature class showing the minimum area that had been surveyed in the deepsea.

During the analysis, the performance rate of the tool was seen to be inversely proportional to the number of input features, as well as the number of list groupings chosen. As an illustration, when a small trial dataset was used, it was significantly noted that the speed of processing took shorter time to generate the result. Overall, performance of the tool was taking about one and a half minutes. This means that the speed of the custom tool would be expected to change depending on number of groupings selected and the size of the dataset among other factors. Table 6 shows the average performance of the tool.

Table 5. Performance results of the ROV Survey Area Generator Tool

\begin{tabular}{|l|l|l|}
\hline Operation & $\begin{array}{l}\text { No. of ROV Observation } \\
\text { Points }\end{array}$ & $\begin{array}{l}\text { Average Performance } \\
\text { Speed (minutes and } \\
\text { seconds) }\end{array}$ \\
\hline $\begin{array}{l}\text { Executing Minimum } \\
\text { Bounding Geometry }\end{array}$ & 216,524 & 2 minutes 35 seconds \\
\hline $\begin{array}{l}\text { Executing Buffer and } \\
\text { Dissolving }\end{array}$ & & 14 minutes 40 seconds \\
\hline & $\mathbf{2 1 6 , 5 2 4}$ & $\mathbf{1 7}$ minutes 15 seconds \\
\hline
\end{tabular}

After running the ROV Survey Area Generator tool in ArcGIS Pro, a total of 2,645 geometry features representing the surveyed sections of the deep-sea were generated. However, it should be noted that, at a global scale, the areas generated were very hard to notice on the map. Figure 6-4 shows a map of the northern section of the Island of Hawaii with mapped areas represented as very minute polygons. This clearly shows that at a very small scale, the features mapped cannot be differentiated from the vast deep-sea. 


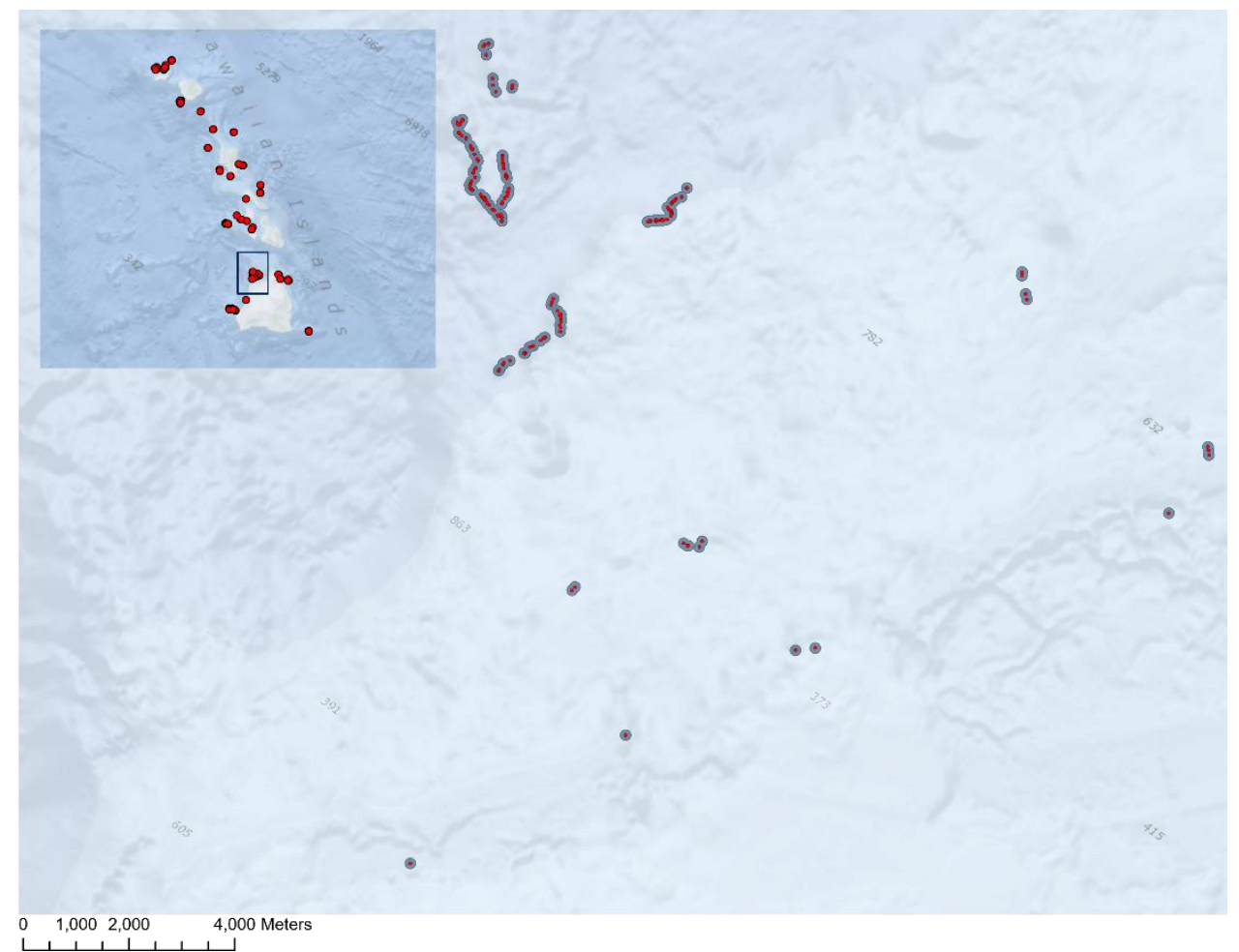

Figure 6-4: Map showing mapped areas in Hawaii

Figure 6-5 shows a section of Monterey Bay zoomed to a scale of 1:400,000. When the features were zoomed in, they could be clearly seen. However, due to the many dives that have been performed in Monterey Bay, it is seen that there are many overlaps of coverages occurring in that section. 


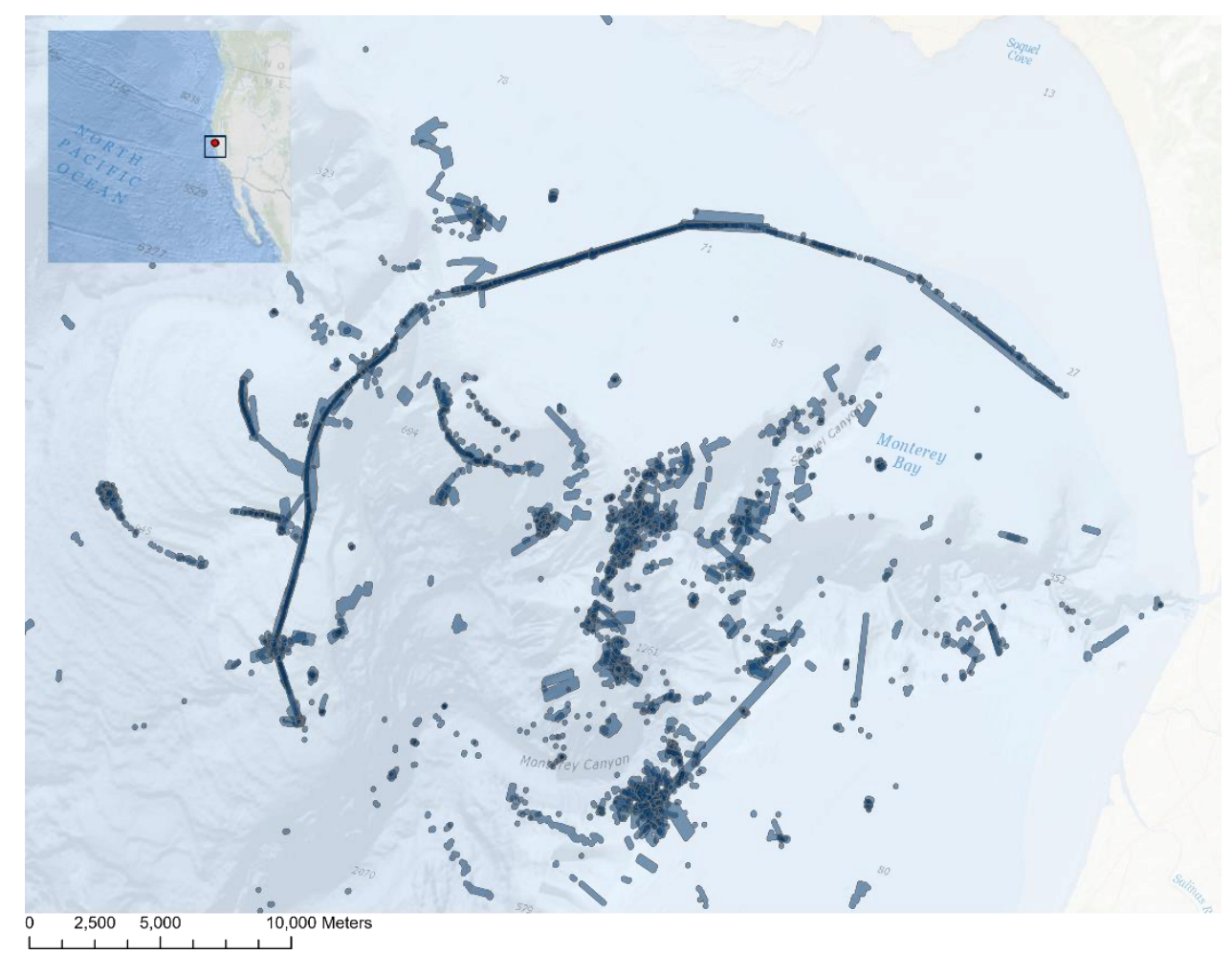

Figure 6-5: Map showing mapped areas in Monterey Bay

\subsection{Representation Complications and Data Issues}

A major part of this project revolved around cleaning and preparation of the dataset. Nothing could have been accomplished without deeply understanding every bit of the dataset. Initially, there were various approaches that seemed to have potential in showing the areas that could have been covered by an ROV in the deep-sea. First, Figure 6-4 shows an approach that aimed at using the Points To Line function from the ArcGIS Pro toolbox. However, this was limited to specifying only one field as the unique line field, and thus led to lines drawing all over the ocean space and other lines coming from the same points more than once as shown in the map. Some lines drew from Hawaii all the way to Monterey. Secondly, Figure 6-5 shows the use of Aggregate Points tool to make square polygon features. This tool returned a polygon of tiles measuring 500 meters by 500 meters, as specified in the input parameter of the tool, with count of points as well as optional statistics at all locations where dive points occured. After discussing this approach with the client, we realized that the Aggregate Points approach was not a good fit for our case. If each tile was partly touched by a single observation, it would mean that the area had been covered, even though partially. This would fit where there is a need to show the areas that have received adequate exploration and those that have not. A superimposition of the two discussed methods are highlighted in Figure 6-6 while Figure 6-7 shows the Minimum Bounding Geometry approach that was adopted for this project case. Table 8 shows the surveyed areas calculated for both the Aggregate Points and Minimum Bounding Geometry approaches. Note that the Points To Line approach is not included since we never went far beyond creating a buffer of the lines produced. 


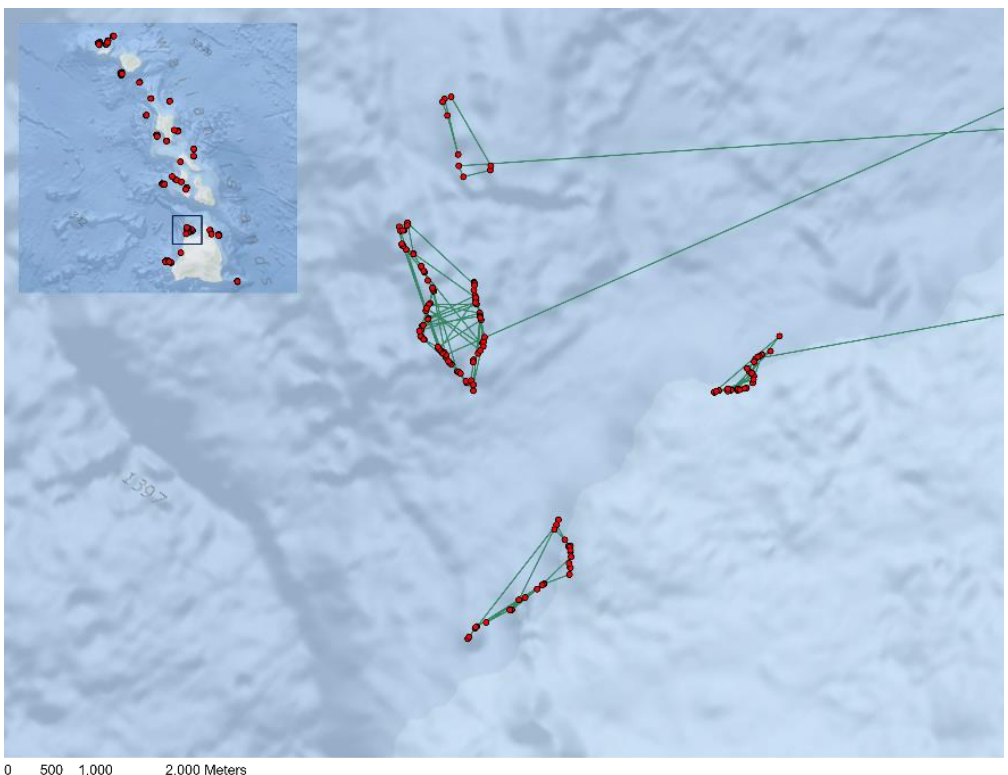

$\begin{array}{lll}500 & 1,000 \quad 2,000 \text { Meters }\end{array}$

Figure 6-6: Using the Points To Line Approach

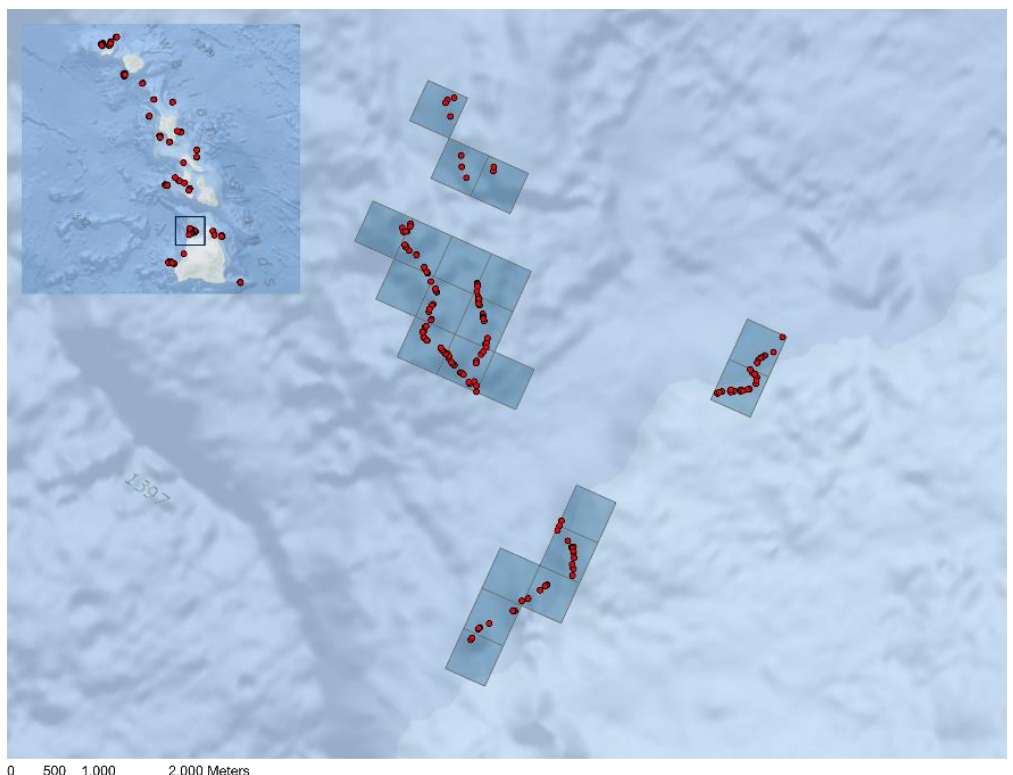

Figure 6-7: Using the Aggregate Points approach 


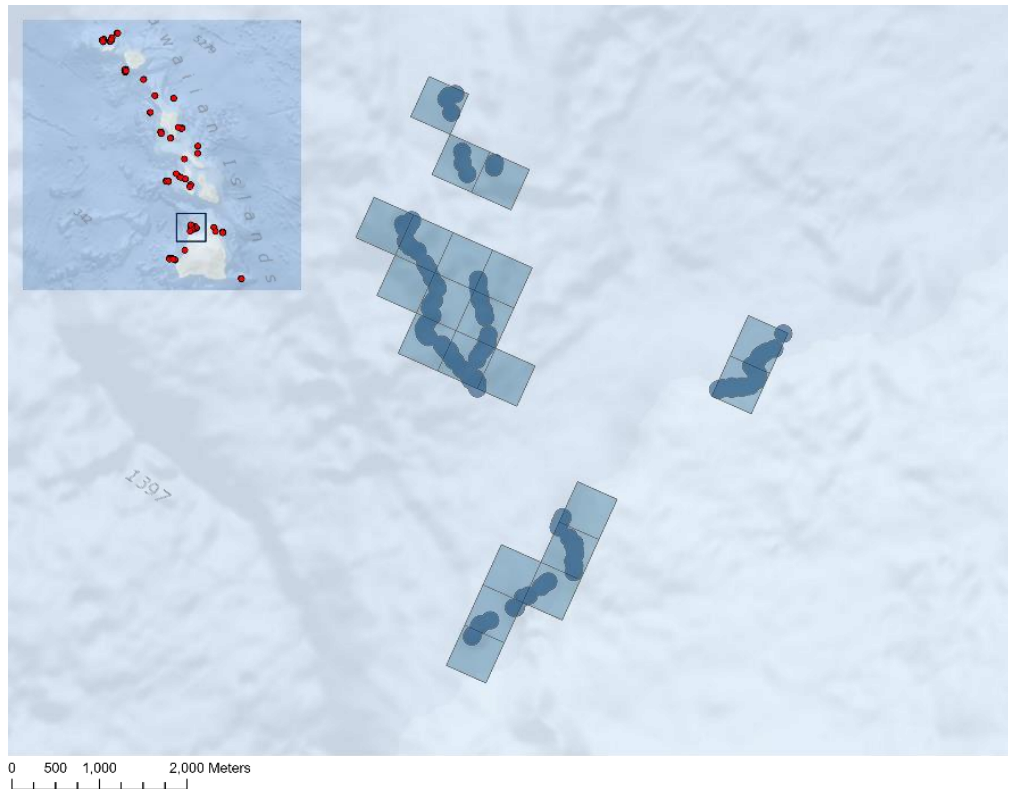

Figure 6-8: Superimposition of Minimum Bounding Geometry and Aggregate Points approaches

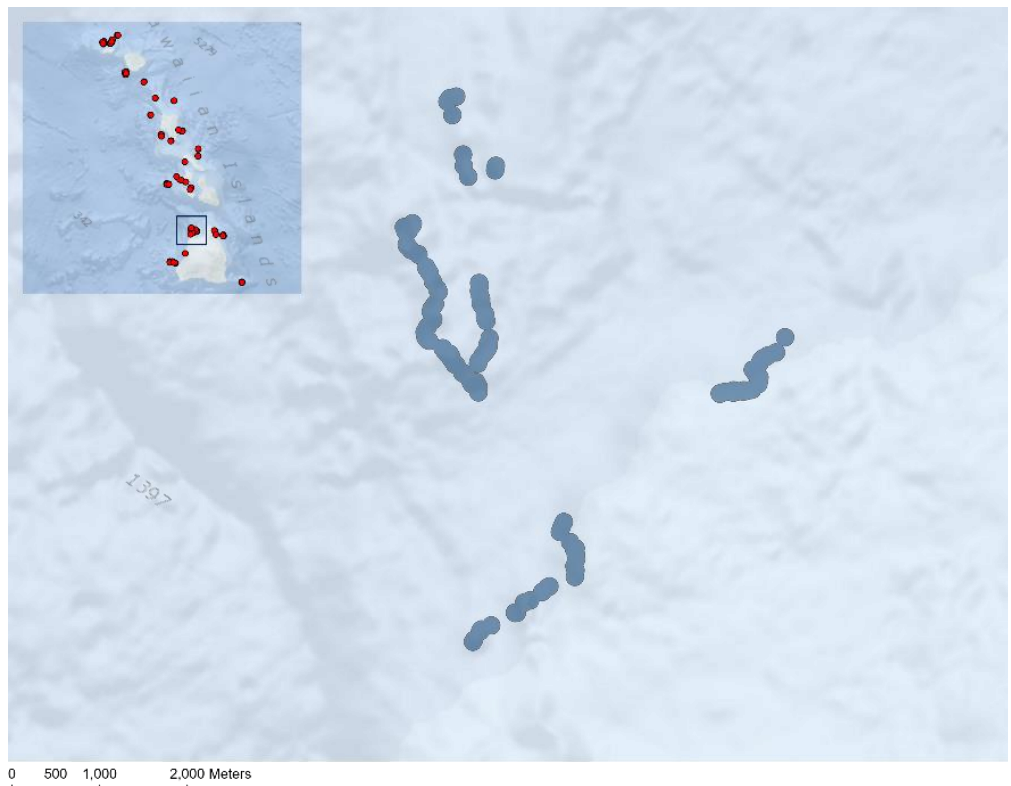

Figure 6-9: Using the Minimum Bounding Geometry approach 
Table 6. Mapped Areas of ROV using the ROV Survey Area Generator Tool and Aggregate Points in GeoAnalytics Desktop toolbox approaches

\begin{tabular}{|l|l|l|l|l|}
\hline \multirow{2}{*}{$\begin{array}{l}\text { Deep sea } \\
\text { Section }\end{array}$} & \multicolumn{2}{|c|}{ No. of Features/bins } & \multicolumn{2}{c|}{ Covered Areas $\left(\mathbf{m}^{2}\right)$} \\
\cline { 2 - 5 } & $\begin{array}{l}\text { ROV Survey } \\
\text { Area } \\
\text { Generator } \\
\text { Tool }\end{array}$ & $\begin{array}{l}\text { Aggregate } \\
\text { Points } \\
\text { Tool }\end{array}$ & $\begin{array}{l}\text { ROV Survey Area } \\
\text { Generator Tool }\end{array}$ & $\begin{array}{l}\text { Aggregate } \\
\text { Points Tool }\end{array}$ \\
\hline Monterey Bay & 52 & 3032 & $397,179,209$ & $758,000,000$ \\
\hline Hawaii & 2,221 & 167 & $9,959,700$ & $41,750,000$ \\
\hline Total & $\mathbf{2 , 2 7 3}$ & $\mathbf{3 1 9 9}$ & $\mathbf{4 0 7 , 1 3 8 , 9 0 9}$ & $\mathbf{7 9 9 , 7 5 0 , 0 0 0}$ \\
\hline
\end{tabular}

\subsection{Summary}

The design and implementation of the project worked towards helping achieve the goals and objectives of the study. This chapter discussed the major use cases of the solution of the project which was the discussion of the results and analysis of the ROV Survey Area Generator tool. The chapter also gave a highlight of the methods for representing ROV mapped areas that could have been used and the reason why each proved not to be workable in helping to achieve the objective of the project. 



\section{Chapter 7 - Conclusions and Future Work}

Since this study on Spatial Representation of NOAA's Remotely Operated Vehicles Dive Tracks had a defined scope, spatially representing the discrete ROV dive track surveys as polygons, it was designed to achieve the objectives within the available time. This chapter revisits the major tasks that were carried out for this project. Section 7.1 gives an overview of the project while section 7.2 and 7.3 documents the future work and the summary respectively.

\subsection{Overview}

Initially, it was stated in chapter 1 that the objective of this study was to help the client, the Deep-Sea Coral Research and Technology Program (DSCRTP), create a spatial representation of the area covered by each ROV while searching for corals and sponges using the Okeanos ship explorer. In order to achieve this objective, the required tools in ArcGIS Pro, Minimum Bounding Geometry and Buffer tools, had to be customized.

During the design phase of the project, the data was cleaned so that the analysis could be done. However, even with cleaning of the dataset, it almost became impossible to produce a reasonable result. The coral and sponge dataset supplied by the client needed to have a defined way of grouping the dives so that a perfect representation of coverages could be made. After closely studying the dataset and through the help of the client, six fields were decided to be ideal for grouping the dives. This grouping information can be found in more detail in Chapter 5, which discusses the implementation of the project.

Once the fields were defined, the best suitable tool to use within ArcGIS Pro version 2.4.1 was found to be the Minimum Bounding Geometry. This tool had the capability to allow the user to define a grouping option based on the input features. The process that followed involved customizing this tool to suit this project in producing the ROVs coverage areas.

\subsection{Future Research}

As indicated in the previous chapters, the most critical part of this project was to make sure that the dataset had the best grouping of each dive. This would allow the dives with the same event identity, but were collected in different time periods and locations, to be grouped uniquely. Since the Deep-Sea Coral Research and Technology Program (DSCRTP) continues to search and collect information for corals and sponges using the Okeanos ship explorer, varying amounts of information that are available from different ROV surveys used will always be recorded. Therefore, it would be advisable to define a better method of extrapolating the ROV survey tracks. As a result, this study would like to suggest possible future GIS work to devise a better method of extrapolating ROV survey tracks. This would ensure that a standard method of representing the varied information from different ROVs, not only those used by NOAA ship Okeanos, but also from other places in the world, is used in the future when a survey is conducted. 


\subsection{Summary}

The major goal of this project was to show the areas that have been covered during the search for corals and sponges. This information on coverages would be vital for the Regional fisheries management and other resource managers who determine rules governing where, when, and how people can fish the deep sea, and they have the power to protect corals and sponges. Therefore, this project provided a valuable contribution for deep-sea coral and sponge management and protection. Spatially representing covered areas to accounting for a measure of survey effort would help put the number of animals found in each location into a more comparable context. This critical information would also help researchers and managers understand where we have looked and not found corals and sponges, and where we have yet to survey in the deep sea. 


\section{Works Cited}

About Us-NOAA's Deep-Sea Coral Data Portal. (2017). [Page]. NOAA's Deep-Sea Coral Data Portal. Retrieved September 19, 2019, from https://deepseacoraldata.noaa.gov/about-us

Cadenas, O., \& Megson, G. M. (2019). Preprocessing 2D data for fast convex hull computations. PLOS ONE, 14(2), e0212189. https://doi.org/10.1371/journal.pone.0212189

Grunbaum, B., \& Shephard, G. C. (1977). Tilings by Regular Polygons. Mathematics Magazine, 50(5), 227-247. https://doi.org/10.2307/2689529

Henry, L.-A., \& Roberts, J. M. (2007). Biodiversity and ecological composition of macrobenthos on cold-water coral mounds and adjacent off-mound habitat in the bathyal Porcupine Seabight, NE Atlantic. Deep Sea Research Part I: Oceanographic Research Papers, 54(4), 654-672.

Hogg, M. M., Tendal, O. S., Conway, K. W., Pomponi, S. A., Van Soest, R. W. M., Gutt, J., Krautter, M., \& Roberts, J. M. (2010). Deep-seas Sponge grounds: Reservoirs of biodiversity.

Hourigan et al. - The State of Deep-Sea Coral and Sponge Ecosystems .pdf. (n.d.).

Hourigan, T. F., Etnoyer, P. J., \& Cairns, S. D. (n.d.). The State of Deep-Sea Coral and Sponge Ecosystems of the United States. 481.

Hourigan, T. F., Etnoyer, P. J., \& Cairns, S. D. (2017). The state of deep-sea coral and sponge ecosystems of the United States. US Department of Commerce, National Oceanic and Atmospheric Administration ....

Hourigan, T. F., Etnoyer, P. J., McGuinn, R. P., Whitmire, C. E., Dorfman, D. S., Dornback, M., Cross, S. L., \& Sallis, D. E. (2015). An introduction to NOAA's National Database for Deep-Sea Corals and Sponges. https://doi.org/10.7289/v5/tm-nos-nccos-191

Hovland, M. (2008). Deep-Water Coral Reefs. Unique Biodiversity Hot-Spots. https://doi.org/10.1007/978-1-4020-8460-7

Jamieson, A. (2015). The Hadal Zone: Life in the Deepest Oceans. Cambridge University Press.

Leal, M. C., Puga, J., Serôdio, J., Gomes, N. C., \& Calado, R. (2012). Trends in the discovery of new marine natural products from invertebrates over the last two decades-where and what are we bioprospecting? PLoS One, 7(1), e30580.

Mehbub, M., Lei, J., Franco, C., \& Zhang, W. (2014). Marine sponge derived natural products between 2001 and 2010: Trends and opportunities for discovery of bioactives. Marine Drugs, 12(8), 4539-4577.

Mumby, P. J., Green, E. P., Edwards, A. J., \& Clark, C. D. (1997). Coral reef habitat mapping: How much detail can remote sensing provide? Marine Biology, 130(2), 193-202. https://doi.org/10.1007/s002270050238

Neves, B. M., Du Preez, C., \& Edinger, E. (2014). Mapping coral and sponge habitats on a shelf-depth environment using multibeam sonar and ROV video observations: Learmonth Bank, northern British Columbia, Canada. Deep Sea Research Part II: Topical Studies in Oceanography, 99, 169-183. https://doi.org/10.1016/j.dsr2.2013.05.026

Osundwa, J. (2016). Development of a Coral Reef Mapping Tool for St. Maarten. 
Parrish, F. A., Baco, A. R., Lumsden, S. E., Hourigan, T. F., Bruckner, A. W., \& Dorr, G. (2007). The state of deep coral ecosystems in the United States.

Peng Dong, Chongjun Yang, Xiaoping Rui, Liqiang Zhang, \& Qimin Cheng. (2003). An effective buffer generation method in GIS. IGARSS 2003. 2003 IEEE

International Geoscience and Remote Sensing Symposium. Proceedings (IEEE Cat. No.03CH37477), 6, 3706-3708 vol.6. https://doi.org/10.1109/IGARSS.2003.1295244

van Kreveld, M. (2006). Computational geometry: Its objectives and relation to GIS. Nederlandse Commissie Voor Geodesie (NCG), 1-8.

Zhou, G., Huang, S., Wang, H., Zhang, R., Wang, Q., Sha, H., Liu, X., \& Pan, Q. (2018). A Buffer Analysis Based on Co-Location Algorithm. International Archives of the Photogrammetry, Remote Sensing and Spatial Information Sciences, 42, 3. 


\section{Appendix A. Project Models}

Add Event Number Model

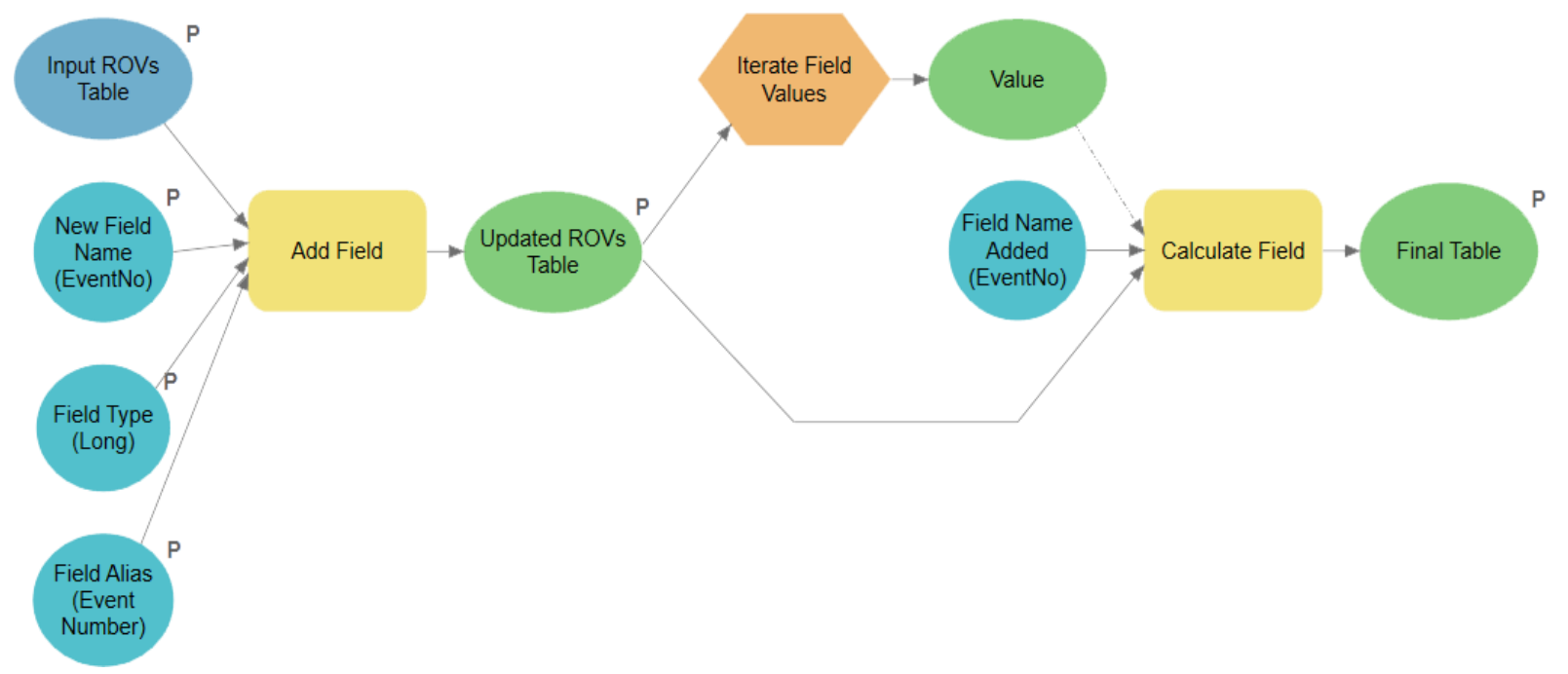

ROV Survey Area Generator Model

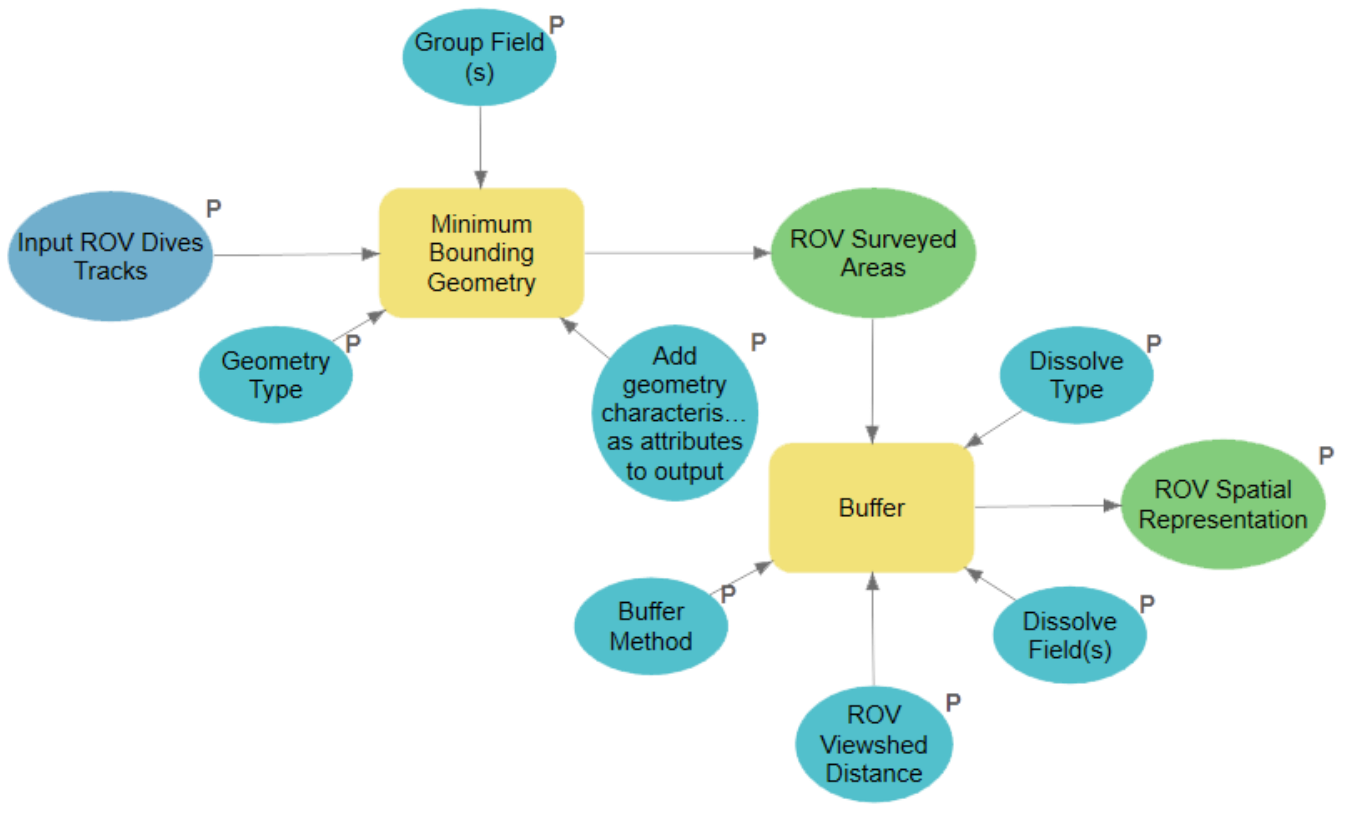




\section{Appendix B. List of Grouping Fields for the Remotely Operated Vehicles Observations}

These fields are established and defined by the Deep-Sea Corals Research and Technology Program in their data dictionary.

\begin{tabular}{|l|l|}
\hline Field Name & Description \\
\hline LargeMarineEcosystem & Large Marine Ecosystem using set designations \\
\hline Country & $\begin{array}{l}\text { Country in whose Exclusive Economic Zone the } \\
\text { observation or sample was collected, based on } \\
\text { Exclusive Economic Zones Boundaries (EEZ) }\end{array}$ \\
\hline ObservationYear & The year that the observation was made \\
\hline SampleID & $\begin{array}{l}\text { The primary identification label or code of the } \\
\text { specimen or catch record exactly as reported by the } \\
\text { Data Provider. }\end{array}$ \\
\hline EventNo & $\begin{array}{l}\text { ID number of the survey event (e.g., dive number, } \\
\text { transect, or trawl haul number) on which the } \\
\text { sample was made. }\end{array}$ \\
\hline
\end{tabular}

